\title{
Is chronic inhibition of phosphodiesterase type 5 cardioprotective and safe? A meta-analysis of randomized controlled trials
}

Elisa Giannetta ${ }^{1}$, Tiziana Feola ${ }^{1}$, Daniele Gianfrilli ${ }^{1}$, Riccardo Pofi ${ }^{1}$, Valentina Dall'Armi ${ }^{2}$, Roberto Badagliacca ${ }^{3}$, Federica Barbagallo ${ }^{1}$, Andrea Lenzi $^{1}$ and Andrea M Isidori ${ }^{*}$

\begin{abstract}
Background: The myocardial effects of phosphodiesterase type 5 inhibitors (PDE5i) have recently received consideration in several preclinical studies. The risk/benefit ratio in humans remains unclear.

Methods: We performed a meta-analysis of randomized, placebo-controlled trials (RCTs) to evaluate the efficacy and safety of PDE5i on cardiac morphology and function. From March 2012 to December 2013 (update: May 2014), we searched English-language studies from MEDLINE, EMBASE, Cochrane Central Register of Controlled Trials and SCOPUS-selecting RCTs of continuous PDE5i administration that reported cardiovascular outcomes: cardiac geometry and performance, afterload, endothelial function and safety. The pooled estimate of a weighted mean difference between treatment and placebo was obtained for all outcomes using a random effects model. A test for heterogeneity was performed and the $\mathrm{I}^{2}$ statistic calculated.

Results: Overall, 1,622 subjects were treated, with 954 randomized to PDE5i and 772 to placebo in 24 RCTs. According to our analysis, sustained PDE5 inhibition produced: (1) an anti-remodeling effect by reducing cardiac mass $(-12.21$ $\mathrm{g} / \mathrm{m}^{2}, 95 \%$ confidence interval $\left.(\mathrm{Cl}):-18.85 ;-5.57\right)$ in subjects with left ventricular hypertrophy (LVH) and by increasing end-diastolic volume $\left(5.00 \mathrm{~mL} / \mathrm{m}^{2} ; 95 \% \mathrm{Cl}: 3.29 ; 6.71\right)$ in non-LVH patients; (2) an improvement in cardiac performance by increasing cardiac index $\left(0.30 \mathrm{~L} / \mathrm{min} / \mathrm{m}^{2}, 95 \% \mathrm{Cl}\right.$ : 0.202; 0.406) and ejection fraction (3.56\%, 95\% Cl: 1.79; 5.33). These effects are parallel to a decline of N-terminal-pro brain natriuretic peptide (NT-proBNP) in subjects with severe LVH (-486.7 pg/ml, 95\% Cl: -712; -261). PDE5i administration also produced: (3) no changes in afterload parameters and (4) an improvement in flow-mediated vasodilation (3.31\%, 95\% Cl: 0.53; 6.08). Flushing, headache, epistaxis and gastric symptoms were the commonest side effects.

Conclusions: This meta-analysis suggests for the first time that PDE5i have anti-remodeling properties and improve cardiac inotropism, independently of afterload changes, with a good safety profile. Given the reproducibility of the findings and tolerability across different populations, PDE5i could be reasonably offered to men with cardiac hypertrophy and early stage heart failure. Given the limited gender data, a larger trial on the sex-specific response to long-term PDE5i treatment is required.
\end{abstract}

Keywords: Phosphodiesterase-5, Heart failure, Hypertension, Endothelial function, Cardiac remodeling, Cardiac hypertrophy, Erectile dysfunction, Sildenafil, Pulmonary hypertension

\footnotetext{
* Correspondence: andrea.isidori@uniroma1.it

'Department of Experimental Medicine, Sapienza University of Rome, Viale del Policlinico 155, Rome 00161, Italy

Full list of author information is available at the end of the article
} 


\section{Background}

For many years, the use of selective phosphodiesterase type 5 inhibitors (PDE5i) has been limited to on-demand administration for erectile dysfunction (ED) [1]. These drugs have recently been shown to affect hemodynamics, right heart performance and oxygenation in patients with pulmonary arterial hypertension (PAH) [2-4]. In addition, PDE5i are currently used in continuous administration for rehabilitation of erectile function and relief of lower urinary tract symptoms [5], although their systemic effects and safety have not been formally appraised.

Initial studies on PDE5i investigated their possible use in the symptomatic relief of angina. However, this was soon abandoned due to the risk of coronary steal or hypotension. Nevertheless, the potential role of PDE5i in non-urological fields continued to attract interest [6,7]. In parallel, several preclinical experimental studies demonstrated that PDE5i were beneficial in ischemia/reperfusion injury, myocardial infarction, doxorubicin-induced cardiotoxicity, hypertrophic cardiac remodeling and heart failure (HF) [8,9]. All these conditions share an enhanced expression of PDE5 enzyme in cardiomyocytes.

Despite these promising animal data, the cardioprotective effect of PDE5i in humans remained unclear. Of note is the discrepancy between the amount of solid experimental data and the paucity of translational studies for drugs that are readily available and widely used with other indications. The few human studies primarily targeting specific cardiac conditions - postmyocardial infarction diastolic dysfunction, diabetic cardiomyopathy, mild and severe heart failure [10-13] suggest a potential effect of PDE5i on cardiac kinetics, geometry and performance, although not universally [14]. Controversy also exists concerning the claimed mechanisms: some hypothesized a direct effect within cardiomyocytes [11,12], some suggested coronary [15] and peripheral vasodilation [16] and yet others a peripheral endothelial effect [17]. However, a much larger number of trials is available reporting cardiac outcomes as secondary endpoints after continuous PDE5i administration.

We performed this meta-analysis to investigate whether chronic PDE5 inhibition modulates cardiac parameters in different clinical settings, exploring both cardiac and peripheral vascular effects, since these endpoints have never been fully assessed in a comprehensive review of the literature.

Specially, we aimed to answer the following questions:

'Does chronic administration of PDE5i produce clinically meaningful changes in cardiac remodeling and performance?', 'Does chronic administration of PDE5i produce hemodynamic and/or endothelial function changes?', 'Is chronic PDE5i administration well-tolerated and safe?'

\section{Methods}

We performed this study according to the Cochrane Collaboration and PRISMA statement [18].

\section{Data sources and searches}

From March 2012 to December 2013 we searched for English-language articles in MEDLINE, EMBASE, Cochrane Library and SCOPUS. Search terms were: sildenafil/tadalafil/vardenafil/PDE5i AND hypertension/ blood pressure/pulmonary hypertension/cardiovascular disease/ heart/heart failure/endothelium/endothelial function. We updated the search in May 2014, but no further studies were included.

\section{Study selection}

Eligibility criteria for study selection included: 1) randomized placebo-controlled trials (RCTs); and 2) chronic PDE5i administration defined as a continuous, daily or alternate day (for tadalafil only based on its half-life), prolonged ( $\geq 4$ weeks) administration. We selected studies reporting any cardiovascular outcomes (as primary or secondary endpoint) independently of the baseline characteristics of the study population.

We excluded reviews, editorials, commentaries, letters, non-RCTs, animal studies, co-administration of PDE5i with other therapies (that is, endothelin antagonist or bosentan) or measuring outcomes under stress or under hypoxic conditions.

Three independent reviewers evaluated all selected titles and abstracts, and for articles considered potentially eligible, full text reports were considered. Interobserver agreement was high (98\%: 434/441 RCTs chosen for full text analysis). Where disagreement occurred, a unanimous decision was taken after open discussion. Figure 1 shows the literature eligibility assessment process.

\section{Data extraction and quality assessment}

Two reviewers (T.F. and R.P.) independently extracted data on study design, sample population (age, gender, clinical status, comorbidities) and treatment characteristics (active compound: sildenafil, tadalafil, vardenafil; dosage and length of treatment). Table 1 summarizes the features of selected studies [see also Additional file 1: Table S1].

In order to compare all studies on the effect of the treatment at the end of the planned therapeutic cycle, we excluded ad interim data, and only end-of-treatment values were recorded. The third investigator (E.G.) performed quality control checks on extracted data. Risk of bias for all trials was independently assessed by the investigators, using the Cochrane risk-of-bias algorithm [19] [see Additional file 1: Table S2]. 


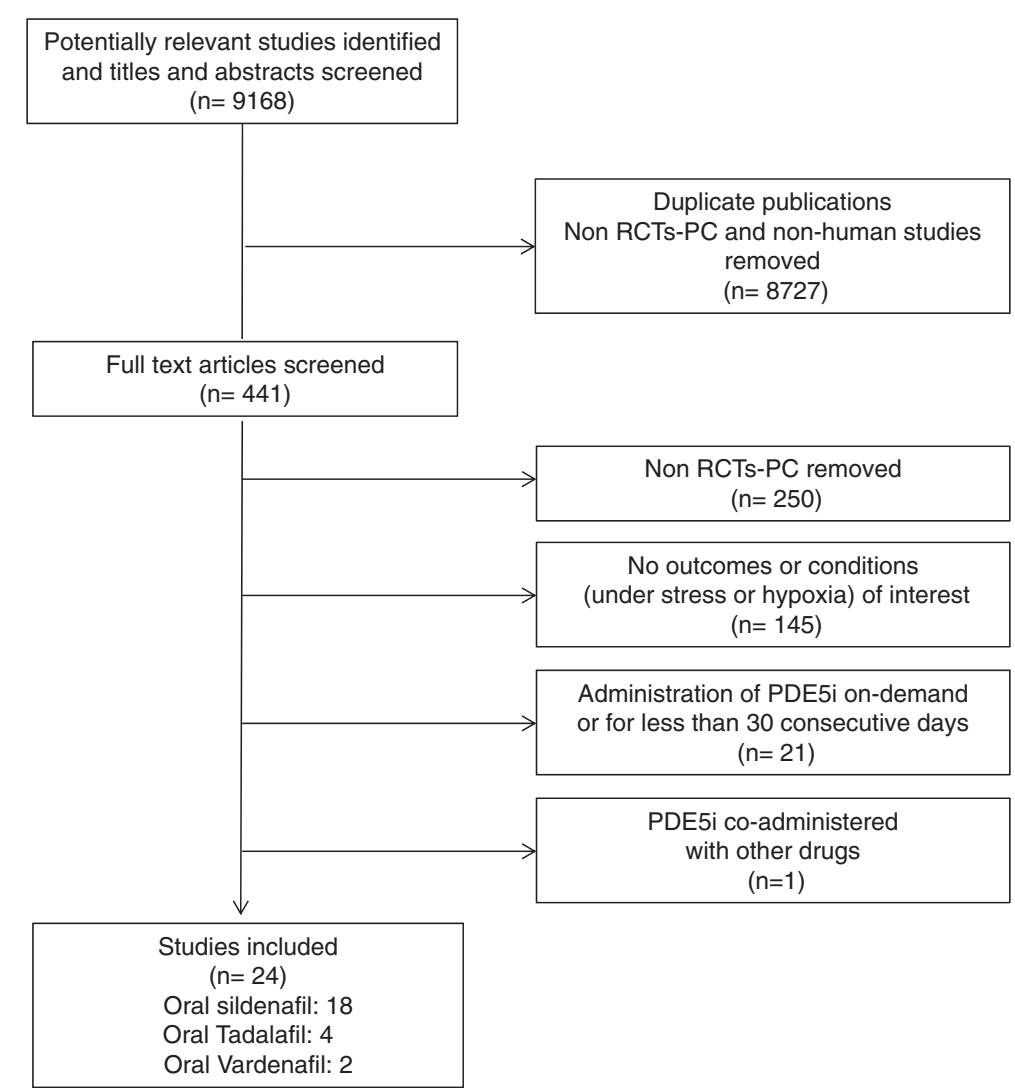

Figure 1 Study flow diagram. PDE5i: phosphodiesterase 5-inhibitors; RCTs: randomized placebo-controlled trials.

\section{Outcomes}

Selected treatment efficacy outcomes were: cardiac geometry (left ventricular mass index: LVMi, end-diastolic volume index: EDVi, interventricular septum: IVS, ventricular transverse diameter: VTD), cardiac performance (cardiac index; ejection fraction: EF; the ratio of the early -E- to late -A- ventricular filling velocities: E/A ratio), neuroendocrine biomarkers (NT-proBNP) and hemodynamic/endothelial parameters (heart rate: HR, blood pressure: BP, systemic vascular resistance index: SVRi, flow mediated vasodilation: FMD). Information on adverse events was extrapolated and analyzed to investigate treatment safety.

\section{Data synthesis and analysis}

Quantitative data extracted from the papers for all treatment efficacy outcomes were baseline and after treatment/placebo means \pm standard deviation (SD). When differences from baseline (means $\pm \mathrm{SD}$ ) were reported, these were also extracted. When summary statistics were not adequately or fully reported (for example, missing pre-post treatment mean difference \pm SD on a specific outcome; standard error of an estimated effect and no corresponding SD), these were calculated whenever possible. When baseline levels, post-treatment and/or change from baseline data were missing or inconsistent, the authors of the original papers were contacted in order to obtain the necessary information [see Additional file 1: Statistical Analysis]. Several studies (16/24) reported data on mixed populations, male and female; however, only 1 of the 16 contacted authors provided raw data separately [20], thus it was not possible to stratify by gender.

In order to examine the efficacy of PDE5i in the different clinical settings, all retrieved studies were categorized a priori according to the following categories: 1) moderate-severe left ventricular hypertrophy (LVH) versus non/mild-LVH (based on LVMi values above or below $131 \mathrm{~g} / \mathrm{m}^{2}$ [21] and where not available, on NTproBNP levels above or below $700 \mathrm{pg} / \mathrm{mL}$ ) [22]; 2) left versus right heart disease; 3) cardiac disease versus non-cardiac disease; 4) HF with reduced EF versus HF with preserved EF; 5) age: younger versus older than 60 years of age; and 6) active compound: sildenafil versus tadalafil versus vardenafil. The same categories were also used for the subgroup analysis. A minimum of two studies were used for subgroup analyses; however, findings stemming from such analyses were interpreted with care. Where a specific subgroup involved a single study, as occurred for HF with preserved EF [14], the analysis was not performed. 
Table 1 Characteristics of studies selected for analysis

\begin{tabular}{|c|c|c|c|c|c|c|}
\hline Study & Design & Condition & Treatment & Dose and time & $\begin{array}{l}\text { Number of } \\
\text { patients (PP) }\end{array}$ & $\begin{array}{l}\text { Mean age (SD) or } \\
\text { age range }\end{array}$ \\
\hline \multirow{3}{*}{ Aldashev AA, Thorax, 2005 [26] } & \multirow{2}{*}{$R, D B, P C$} & \multirow{2}{*}{$\mathrm{HAPH}$} & \multirow{2}{*}{ S } & $25 \mathrm{mg} \times 3 /$ die & $9 S$ versus $8 P$ & \multirow{2}{*}{$61(8)$} \\
\hline & & & & $100 \mathrm{mg} \times 3 /$ die 12 weeks & $5 S$ versus $8 P$ & \\
\hline & \multirow{3}{*}{$R, D B, P C$} & \multirow{3}{*}{ HFrEF } & \multirow{3}{*}{ S } & $25 \mathrm{mg} \times 2 /$ die $->2$ weeks & & \multirow{2}{*}{ S: 51.29 (14.80) } \\
\hline \multirow[t]{2}{*}{ Amin A, Congest Heart Fail, 2013 [38] } & & & & Then & $53 \mathrm{~S}$ versus $51 \mathrm{P}$ & \\
\hline & & & & $50 \mathrm{mg} \times 3 /$ die $->10$ weeks & & P: 50.61 (4.18) \\
\hline \multirow{2}{*}{ Andersen MJ, Circulation, 2013 [10] } & \multirow{2}{*}{$R, D B, P C$} & \multirow{2}{*}{ Diastolic Dysfunction after Ml } & \multirow{2}{*}{ S } & \multirow{2}{*}{$40 \mathrm{mg} \times$ 3/die 9 weeks } & \multirow{2}{*}{$34 \mathrm{~S}$ versus $33 \mathrm{P}$} & S: 63 (8) \\
\hline & & & & & & P: 62 (7) \\
\hline \multirow{4}{*}{ Badesch, J Rheumatol, 2007 [27] } & \multirow{4}{*}{$R, D B, P C$} & \multirow{4}{*}{ PAH-CTD } & \multirow{4}{*}{$\mathrm{S}$} & $20 \mathrm{mg} \times 3 /$ die & $21 \mathrm{~S}$ & S: 52 (15) \\
\hline & & & & $40 \mathrm{mg} \times 3 /$ die & $20 \mathrm{~S}$ & $50(15)$ \\
\hline & & & & $80 \mathrm{mg} \times 3 /$ die & $19 S$ & $54(14)$ \\
\hline & & & & 12 weeks & $22 \mathrm{P}$ & P: 56 (14) \\
\hline \multirow{2}{*}{ Behling A, J Cardiac Fail, 2008 [23] } & \multirow{2}{*}{$\mathrm{R}, \mathrm{DB}, \mathrm{PC}$} & \multirow{2}{*}{$\mathrm{CHF}$} & \multirow{2}{*}{ S } & $50 \mathrm{mg} \times 3 /$ die & \multirow{2}{*}{$11 \mathrm{~S}$ versus $8 \mathrm{P}$} & S: 45 (12) \\
\hline & & & & 4 weeks & & P: 53 (11) \\
\hline \multirow{2}{*}{ Bharani A, Indian Heart J, 2007 [28] } & $R, D B, P C$ & \multirow{2}{*}{$\begin{array}{l}\text { PAH due to congenital left } \\
\text { to right shunt }\end{array}$} & \multirow{2}{*}{ T } & $20 \mathrm{mg} \times 3$ & \multirow{2}{*}{$8 \mathrm{~T}-\mathrm{P}$} & $28(9.38)$ \\
\hline & CO (wo:2 wks) & & & 4 weeks & & \\
\hline Bocchio M, Atherosclerosis, 2008 [33] & $R, D B, P C$ & ED VRF & $\mathrm{T}$ & 20 mg every other day & $18 \mathrm{~T}$ versus $18 \mathrm{P}$ & T: 52.05 (8.98) \\
\hline & Ni, 60,1 , & Le vili & & 12 weeks & & P: 49.61 (12.68) \\
\hline & & & & $20 \mathrm{mg} \times 3 /$ die & $65 \mathrm{~S}$ & S: 47 (14) \\
\hline Galiè N NEJM. 2005 [2] & B. DB PC & PAH & 5 & $40 \mathrm{mg} \times$ 3/die & $63 \mathrm{~S}$ & $51(15)$ \\
\hline & 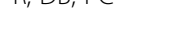 & 年 & J & $80 \mathrm{mg} \times$ 3/die & $65 \mathrm{~S}$ & $48(18)$ \\
\hline & & & & 12 weeks & $65 P$ & P: 49 (17) \\
\hline Giannetta F Girculation 2012 & 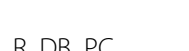 & Diabetic Cardiomyonathy & $\mathrm{C}_{\mathrm{C}}$ & $25+25+50 \mathrm{mg} / \mathrm{die}$ & 20 s inrcus 250 & S: $60.7(7.6)$ \\
\hline 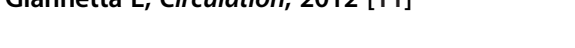 & $n, D D, r C$ & 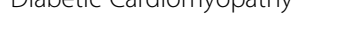 & $\mathrm{J}$ & 3 months & 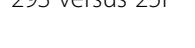 & P: $60.2(8.3)$ \\
\hline Goldberg DJ, Circulation, 2011 [36] & $R, D B, P C$ & CHD after Fontan Operation & s & $20 \mathrm{mg} \times 3 / \mathrm{die}$ & $275-P$ & $149(51)$ \\
\hline & CO (wo:6 wks) & & J & 6 weeks & 21 & (1) \\
\hline Goldberg, DJ Pediatr Cardiol, 2012 [37] & $\mathrm{R}, \mathrm{DB}, \mathrm{PC}$ & CHD after Fontan Operation & s & $20 \mathrm{mg} \times 3 / \mathrm{die}$ & $275-P$ & $149(51)$ \\
\hline & CO (wo:6 wks) & & & 6 weeks & $275-r^{2}$ & (14.9(5.1) \\
\hline & & & & 10 mg/die - >4 weeks & & T: 39.8 (13.1) \\
\hline $\begin{array}{l}\text { Groeneweg G, BMC Muscoloskeletan disorders, } \\
2008[20]\end{array}$ & $\mathrm{R}, \mathrm{DB}, \mathrm{PC}$ & CRPS & $\mathrm{T}$ & Then & $12 \mathrm{~T}$ versus $12 \mathrm{P}$ & $365(106)$ \\
\hline & & & & 20 mg/die - >8 weeks & & P. 30.5 (10.0) \\
\hline Guazzi M, J Am Coll Cardiol, 2007 [24] & $R, D B, P C$ & $\mathrm{CHF}$ & $\mathrm{s}$ & $50 \mathrm{mg} \times 3 /$ die & 235 versus $23 \mathrm{P}$ & S: 62 (3) \\
\hline & & & & 6 months & & P: 63 (4) \\
\hline
\end{tabular}


Table 1 Characteristics of studies selected for analysis (Continued)

\begin{tabular}{|c|c|c|c|c|c|c|}
\hline Guazzi M, Circulation, 2011 [25] & $R, D B, P C$ & HFpEF with PAH & S & $\begin{array}{l}50 \mathrm{mg} \times 3 / \text { die } \\
12 \text { months }\end{array}$ & $22 \mathrm{~S}$ versus $22 \mathrm{P}$ & $53-79$ \\
\hline Guazzi M, Circ Heart Fail, 2011 [12] & $R, D B, P C$ & Systolic HF & $S$ & $\begin{array}{l}50 \mathrm{mg} \times 3 / \text { die } \\
12 \text { months }\end{array}$ & $23 \mathrm{~S}$ versus $22 \mathrm{P}$ & $\begin{array}{l}S: 60(4) \\
P: 61(4)\end{array}$ \\
\hline Guazzi M, Europ J Heart Failure, 2012 [39] & $R, D B, P C$ & $\mathrm{EOB}$ in $\mathrm{HF}$ & S & $\begin{array}{l}50 \mathrm{mg} \times 3 / \text { die } \\
12 \text { months }\end{array}$ & $16 \mathrm{~S}$ versus $16 \mathrm{P}$ & $\begin{array}{l}\text { S: } 66(8) \\
\text { P: } 68(6)\end{array}$ \\
\hline Jing, Z.C, Am J Resp Crit Care Med, 2011 [30] & $R, D B, P C$ & $\mathrm{PAH}$ & V & $\begin{array}{l}5 \mathrm{mg} / \mathrm{die}->4 \text { weeks } \\
\text { then }\end{array}$ & $43 \mathrm{~V}$ versus $16 \mathrm{P}$ & S: $32(12)$ \\
\hline & & & & $5 \mathrm{mg} \times 2 /$ die $->8$ weeks & & P: 29 (8) \\
\hline Lewis GD, Circulation, 2007 [13] & $R, D B, P C$ & Systolic HF and secondary PAH & $S$ & $\begin{array}{l}25 \text { to } 75 \mathrm{mg} \times 3 / \text { die } \\
12 \text { weeks }\end{array}$ & 175 versus $17 \mathrm{P}$ & $\begin{array}{l}\text { S: } 54(4) \\
P: 62(3)\end{array}$ \\
\hline Lewis GD, Circ Heart Fail, 2008 [31] & $R, D B, P C$ & Systolic HF and secondary PAH & $S$ & $\begin{array}{l}25 \text { to } 75 \mathrm{mg} \times 3 / \text { die } \\
12 \text { weeks }\end{array}$ & $15 \mathrm{~S}$ versus $15 \mathrm{P}$ & $\begin{array}{l}\text { S: } 54 \text { (4) } \\
P: 62(3)\end{array}$ \\
\hline Rosano G MC, European Urology, 2005 [34] & $R, D B, P C$ & ED VRF & $\mathrm{T}$ & $\begin{array}{l}20 \text { mg every other day } \\
4 \text { weeks }\end{array}$ & $16 \mathrm{~T}$ versus $16 \mathrm{P}$ & $65.4(6.3)$ \\
\hline Redfield MM, JAMA, 2013 [14] & $R, D B, P C$ & HFpEF & $\mathrm{S}$ & $\begin{array}{l}20 \mathrm{mg} \times 3 / \text { die }->12 \text { weeks } \\
\text { then } 60 \mathrm{mg} \times 3 / \text { die }-> \\
12 \text { weeks }\end{array}$ & $\begin{array}{l}49 S \text { versus } 47 \mathrm{P}^{\mathrm{a}} \\
45 \mathrm{~S} \text { versus } 58 \mathrm{P}^{\mathrm{b}} \\
95 \mathrm{~S} \text { versus } 94 \mathrm{P}^{c}\end{array}$ & $62-77$ \\
\hline Sastry BKS, JACC, 2004 [32] & $\begin{array}{l}R, D B, P C \\
C O(w o: 0)\end{array}$ & $\mathrm{PAH}$ & $S$ & $\begin{array}{l}25 \text { to } 100 \mathrm{mg} \times 3 / \text { die } \\
6 \text { weeks }\end{array}$ & $20 S-P$ & $16-55$ \\
\hline Suntharalingam J, Chest, 2008 [40] & $R, D B, P C$ & CTEPH & $\mathrm{S}$ & $\begin{array}{l}40 \mathrm{mg} \times 3 / \text { die } \\
12 \text { weeks }\end{array}$ & $8 \mathrm{~S}$ versus $10 \mathrm{P}$ & $\begin{array}{l}\text { S: } 49.9(13.1) \\
\text { P: } 60(14.4)\end{array}$ \\
\hline Van AH, J Sex Med, 2005 [35] & $R, D B, P C$ & ED & V & $\begin{array}{l}5 \text { to } 20 \mathrm{mg} / \mathrm{die} \\
12 \text { weeks }\end{array}$ & $175 \mathrm{~V}$ versus $175 \mathrm{P}$ & $\begin{array}{l}V: 22-76 \\
P: 22-78\end{array}$ \\
\hline
\end{tabular}

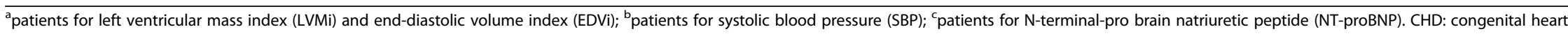
disease; CHF: chronic heart failure; CO: crossover; CRPS: cold complex regional pain syndrome; CTEPH: chronic thromboembolic pulmonary hypertension; DB: double-blind; ED VRF: erectile dysfunction vascular risk factors; EOB: exercise oscillatory breathing; HAPH: high altitude pulmonary hypertension; HF: heart failure; HFpEF: heart failure preserved ejection fraction; HFrEF: heart failure reduced ejection fraction; MI: myocardial infarction; P: placebo; PAH: pulmonary arterial hypertension; PAH-CTD: pulmonary arterial hypertension-connective tissue diseases; PC: placebo-controlled; PP: per protocol analysis; R: randomized; S: sildenafil;

T: tadalafil; V: vardenafil; wks: weeks; wo: washout. 
Adverse events in the treatment group compared to the placebo group were analyzed by relative risks calculated on the intention-to-treat population. Any adverse event found only in one study was not analyzed [see Additional file 1: Statistical Analysis].

A meta-analysis was performed on all outcomes and effect sizes were combined to give a pooled estimate of a weighted mean difference (WMD) between treatment and placebo, the weights being the reciprocals of the variance. A random effects model was fitted. This choice was dictated by the heterogeneity of study characteristics.

The test for heterogeneity was performed and the $\mathrm{I}^{2}$ statistic (low $=30 \%$; moderate $=30 \%$ to $75 \%$; high $\geq 75 \%$ ) calculated. All results are shown in Additional file 1Heterogeneity.

Along with estimation results, forest plots and funnel plots were visually inspected in order to more clearly detect clusters of studies, outlier studies and possible publication biases.

Statistical significance was set at $P<0.05$. The software used for all statistical analysis was STATA/SE V10.

\section{Results}

\section{Study selection}

Figure 1 shows the literature search process in MEDLINE, EMBASE, Cochrane and SCOPUS (March 2012 to December 2013 and updated in May 2014). We identified 9,168 studies as potentially relevant. Of these, 8,727 were excluded based on title and abstract content and 417/441 were excluded after full text analysis due to: non-English language, non-human studies, not RCTs, no outcome of interest, PDE5i co-administered with other drugs. RCTs with PDE5i given on demand or for less than 30 consecutive days were excluded. A total of 24 RCTs were eligible and included in the review (18 administering sildenafil, 4 tadalafil and 2 vardenafil).

\section{Study characteristics}

Table 1 summarizes the 24 reports that met all inclusion criteria. Authors analyzed the effects of continuous PDE5i administration in various cardiac disorders (diastolic dysfunction secondary to myocardial infarction [10], HF [12,14,23-25], PAH [26-32], diabetic cardiomyopathy [11]) and ED [33-35]. One study was performed on a non-cardiological/non-urological condition, enrolling patients with complex regional pain syndrome [20]. Goldberg et al. [36,37] and Lewis et al. [13,31] reported their results in two separate publications, each addressing different endpoints; thus, the 24 retrieved papers described 22 groups of subjects. For parameters reported twice (mean BP, SVR and HR), data from the most recent publications were used [31,37].

Selected trials gave details of 1,622 subjects, with 954 randomized to PDE5i (694 to sildenafil; 54 to tadalafil;
218 to vardenafil), and 772 to placebo (per protocol (PP) analysis), 55 allocated in cross-over trials. Most studies were performed in North America or Europe, with five in Asia [26,28,30,32,38].

The studies varied in terms of: 1) daily dosage: some administered PDE5i with a titration scheme [20,30,38] or based on individual response [14,32,35]; 2) length of treatment: from 4-week to 12-month study periods; 3 ) endpoint assessment method; 4) age; 5) baseline cardiovascular status; and 6) gender: 8 trials enrolled only males [11,12,24,26,33-35,39], and 16 trials a mixed population of 540 females and 459 males (ITT population). All studies were RCTs, double-blind (DB) and placebo-controlled. Four studies were crossover with variable washout periods (from 0 to 6 weeks) [28,32,36,37].

Around half (56\%) of the trials reported cardiovascular data as secondary outcomes. Fourteen trials received funding from pharmaceutical companies (Pfizer, Eli Lilly) $[2,13,20,23,26,30,31,34]$ or foundations $[12,25,36,37,39]$.

\section{Risk of bias}

All publications reported results from RCTs; however, the randomization method and allocation concealment were inappropriately described in $54 \%$ of studies $(13 / 24)$, so the risk of selection bias was unclear for this group, while for the remaining $46 \%$ of trials the risk of selection bias was low.

Regarding the reporting bias, seven studies had high risk and in two additional studies it was unclear. Most studies had a low risk of performance, detection and attrition bias [see Additional file 1: Risk of bias and Table S2].

\section{Synthesis of results}

\section{Efficacy data: PDE-5 inhibitors versus placebo}

Cardiac geometry The following parameters were considered measures of cardiac structure: LVMi, EDVi, IVS and VTD.

LVMi did not change $\left(-4.022 \mathrm{~g} / \mathrm{m}^{2}\right.$; 95\% CI: -10.137 ; 2.093, $P=0.20)$ in the main analysis performed in five trials, all using sildenafil [10-12,14,25] ( $\mathrm{n}=306 ; 72 \%$ male). However, a significant reduction in LVMi (12.207 $\mathrm{g} / \mathrm{m}^{2}$; 95\% CI: -18.846; -5.568, $\left.P<0.001\right)$ was detected in subgroup analysis of patients with left ventricular hypertrophy (Figure 2).

Similarly, the main analysis of EDVi $\left(0.433 \mathrm{~mL} / \mathrm{m}^{2}\right.$; 95\% CI: $-6.49 ; 7.357, P=0.90)$ in four studies $[10-12,14]$ ( $\mathrm{n}=262 ; 71 \%$ male) found no significant change. Subgroup analyses revealed a significant increase in EDVi in non-hypertrophic patients $\left(4.999 \mathrm{~mL} / \mathrm{m}^{2}\right.$; $95 \% \mathrm{CI}$ : 3.286 ; 6.711, $P<0.001, \mathrm{I}^{2}=0.0 \%$ ) (Figure 2 ).

The main analysis of IVS [11,25] and VDT [11,12] was possible in two studies only, with no significant change found (Table 2). 


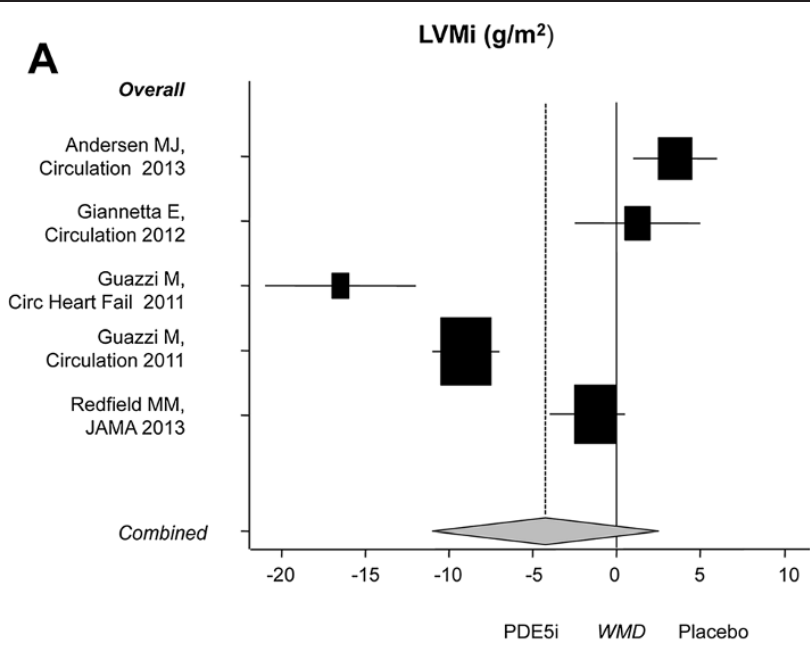

B

$\operatorname{LVMi}\left(\mathbf{g} / \mathrm{m}^{2}\right)$

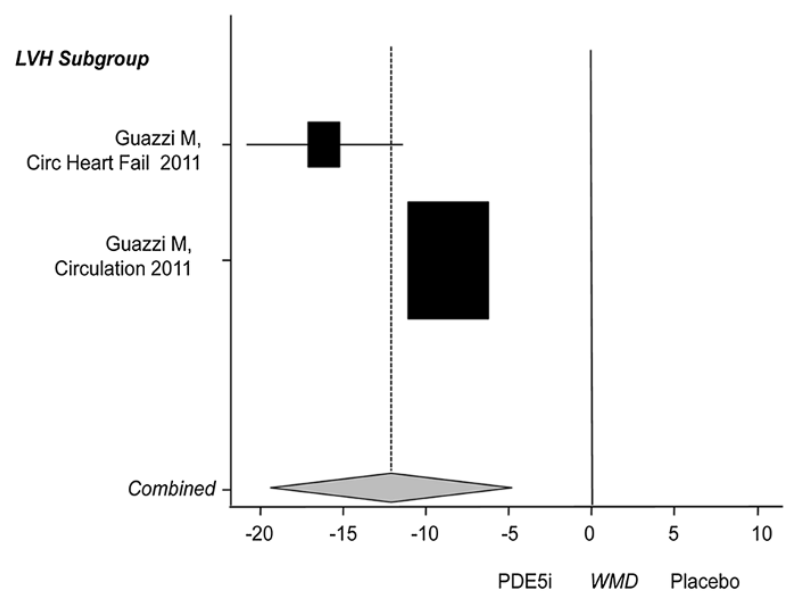

C

$\operatorname{EDVi}\left(\mathrm{ml} / \mathrm{m}^{2}\right)$

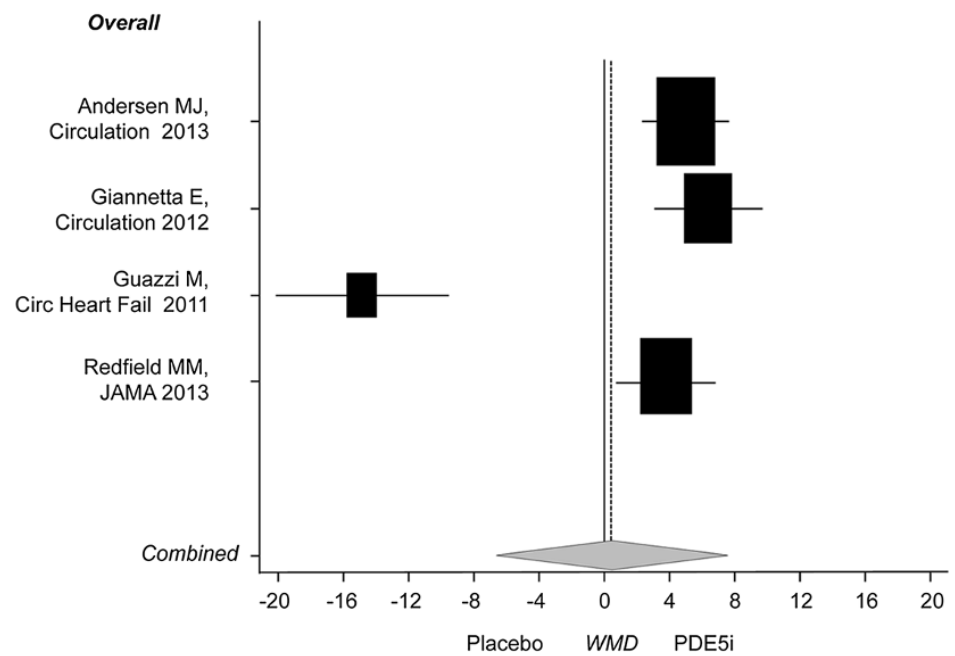

Figure 2 Effects of PDE5i over placebo on cardiac geometry parameters. A) LVMi main analysis; B) LVMi subgroup analysis in patients with $\mathrm{LVH}$; C) EDVi main analysis. Diamond indicates the overall summary estimate for the analysis (width of the diamond represents the $95 \% \mathrm{Cl}$ ); boxes, the weight of individual studies in the pooled analysis. EDVi: end-diastolic volume index; LVH: left ventricular hypertrophy; LVMi: left ventricular mass index; PDE5i: phosphodiesterase type 5 inhibitors. 
Table 2 Results of main analysis and subgroup or sensitivity analyses

\begin{tabular}{|c|c|c|c|c|c|c|c|c|c|c|c|c|c|c|}
\hline Main analysis & $\begin{array}{l}\mathrm{LVMi} \\
\mathrm{g} / \mathrm{m}^{2}\end{array}$ & $\begin{array}{l}\mathrm{EDVi} \\
\mathrm{ml} / \mathrm{m}^{2}\end{array}$ & $\begin{array}{l}\text { IVS } \\
\text { Mm }\end{array}$ & $\begin{array}{l}\text { VTD } \\
\mathrm{mm}\end{array}$ & $\begin{array}{l}\text { Cardiac index } \\
\mathrm{L} / \mathrm{min} / \mathrm{m}^{2}\end{array}$ & EF\% & $\begin{array}{l}\mathrm{E} / \mathrm{A} \\
\text { ratio }\end{array}$ & $\begin{array}{l}\text { NTpro-BNP } \\
\mathrm{pg} / \mathrm{ml}\end{array}$ & HR bpm & $\begin{array}{l}\text { MAP } \\
\mathrm{mmHg}\end{array}$ & $\begin{array}{l}\mathrm{SBP} \\
\mathrm{mmHg}\end{array}$ & $\begin{array}{l}\text { DBP } \\
\mathrm{mmHg}\end{array}$ & $\begin{array}{l}\text { SVRi dynes } \\
\mathrm{m}^{2} / \mathrm{sec} \mathrm{cm}^{-5}\end{array}$ & FMD\% \\
\hline$\overline{\text { WMD }}$ & -4.022 & 0.433 & -0.966 & -2.666 & 0.304 & 3.561 & 0.053 & -161.9 & -0.848 & -0.413 & 0.616 & -0.763 & -88.34 & 3.306 \\
\hline Lower 95\% Cl & -10.137 & -6.49 & -2.230 & -7.145 & 0.202 & 1.786 & -0.275 & -351.5 & -2.398 & -3.023 & -1.113 & -2.174 & -255.75 & 0.530 \\
\hline Upper 95\% Cl & 2.093 & 7.357 & 0.298 & 1.814 & 0.406 & 5.335 & 0.380 & 27.6 & 0.702 & 2.196 & 2.345 & 0.648 & 79.08 & 6.082 \\
\hline - - & - & - & - - & - & - - & - - & - & -- & - - & - & - & - & - & - \\
\hline Number & 306 & 262 & 98 & 99 & 520 & 286 & 116 & 407 & 977 & 355 & 828 & 791 & 232 & 168 \\
\hline$P$ value & 0.197 & 0.902 & 0.134 & 0.243 & $<0.001$ & $<0.001$ & 0.753 & 0.094 & 0.283 & 0.756 & 0.490 & 0.289 & 0.301 & 0.020 \\
\hline I-square & $96 \%$ & $94 \%$ & $95 \%$ & $90 \%$ & $0.0 \%$ & $84 \%$ & $93 \%$ & $82 \%$ & $56 \%$ & $71 \%$ & $49 \%$ & $52 \%$ & $89 \%$ & $99 \%$ \\
\hline References & {$[10-12,14,25]$} & {$[10-12,14]$} & {$[11,25]$} & {$[11,12]$} & $\begin{array}{l}{[2,10,11,25,30,} \\
32,40]\end{array}$ & $\begin{array}{l}{[10-12,24,} \\
25,31,35]\end{array}$ & {$[12,25,37]$} & {$[10-14,40]$} & $\begin{array}{l}{[2,10,11,20,} \\
24,26,28,30 \\
31,35,37,39]\end{array}$ & $\begin{array}{l}{[10,23,25} \\
30,31,38,39]\end{array}$ & $\begin{array}{l}{[10-12,14,20,} \\
24,25,28,35, \\
36,38]\end{array}$ & $\begin{array}{l}{[10-12,20,24-} \\
26,28,35,36,38]\end{array}$ & {$[10,25,30,31,39]$} & {$[11,24,33,34]$} \\
\hline $\begin{array}{l}\text { Subgroup/ } \\
\text { Sensitivity }^{\mathrm{a}}\end{array}$ & $\begin{array}{l}\text { LVMi } \\
\mathrm{g} / \mathrm{m}^{2}\end{array}$ & $\begin{array}{l}\mathrm{EDVi} \\
\mathrm{ml} / \mathrm{m}^{2}\end{array}$ & $\begin{array}{l}\text { IVS } \\
\mathrm{Mm}\end{array}$ & $\begin{array}{l}\text { VTD } \\
\mathrm{mm}\end{array}$ & $\begin{array}{l}\text { Cardiac index } \\
\mathrm{L} / \mathrm{min} / \mathrm{m}^{2}\end{array}$ & EF \% & $\begin{array}{l}\mathrm{E} / \mathrm{A} \\
\text { ratio }\end{array}$ & $\begin{array}{l}\text { NTpro-BNP } \\
\mathrm{Pg} / \mathrm{ml}\end{array}$ & HR bpm & $\begin{array}{l}\text { MAP } \\
\mathrm{mmHg}\end{array}$ & $\begin{array}{l}\text { SBP } \\
\mathrm{mmHg}\end{array}$ & $\begin{array}{l}\text { DBP } \\
\mathrm{mmHg}\end{array}$ & $\begin{array}{l}\text { SVRi dynes } \\
\mathrm{m}^{2} / \mathrm{sec} \mathrm{cm}^{-5}\end{array}$ & FMD \% \\
\hline$\overline{\mathrm{LVH}}$ & -12.207 & & & & 0.082 & 4.382 & 0.053 & -486.7 & 0.829 & 1.156 & 0.135 & -1.936 & 55.63 & 1 \\
\hline Lower 95\% Cl & -18.846 & / & / & / & -0.176 & 2.059 & -0.275 & $-712,8$ & -4.526 & -2.398 & -2.598 & -5.3091 .438 & -277.86 & \\
\hline Upper 95\% Cl & -5.568 & & & & 0.341 & 6.705 & 0.380 & $-260,7$ & 6.185 & 4.710 & 2.867 & - & 389.12 & \\
\hline- & - & & & & - & - & - & -- & -- & - & -- & & - & \\
\hline Number & 89 & & & & 62 & 119 & 116 & 97 & 57 & 74 & 72 & 116 & 74 & \\
\hline$P$ value & $<0.001$ & I & / & / & 0.534 & $<0.001$ & 0.753 & $<0.001$ & 0.761 & 0.524 & 0.923 & 0.261 & 0.744 & / \\
\hline I-square & $88 \%$ & I & / & I & $0.0 \%$ & $79 \%$ & $93 \%$ & $0.0 \%$ & $0.0 \%$ & $0.0 \%$ & $0.0 \%$ & $66 \%$ & $88 \%$ & / \\
\hline Non-LVH & 1.404 & $4.999^{a}$ & & & 0.354 & 0.973 & & -11.20 & 0.691 & & 0.613 & -0.635 & I & / \\
\hline Lower 95\% Cl & -1.908 & 3.286 & / & / & 0.165 & -1.329 & / & -160.8 & -2.330 & / & -5.161 & -9.2788 .008 & & \\
\hline Upper 95\% Cl & 4.717 & 6.711 & & & 0.544 & 3.274 & & 138.4 & 3.713 & & 6.388 & -- & & \\
\hline- & - & - & & & - & - & & - & - & & - & & & \\
\hline Number & 217 & 217 & & & 121 & 121 & & 310 & 121 & & 224 & 121 & & \\
\hline$P$ value & 0.406 & $<0.001$ & / & / & $<0.001$ & 0.408 & I & 0.883 & 0.654 & / & 0.835 & 0.886 & I & / \\
\hline I-square & $80 \%$ & $0.0 \%$ & / & / & $0.0 \%$ & $0.0 \%$ & / & $75 \%$ & $0.0 \%$ & / & $79 \%$ & $91 \%$ & / & I \\
\hline $\begin{array}{l}\text { Left heart } \\
\text { disease }\end{array}$ & -4.022 & 0.433 & -0.966 & -2.666 & 0.298 & 3.561 & 0.053 & $-149.7^{a}$ & 0.651 & -0.951 & $0.079^{a}$ & -1.352 & -70.97 & 2.686 \\
\hline Lower 95\% Cl & $\begin{array}{l}-10.137 \\
2.093\end{array}$ & $\begin{array}{l}-6.49 \\
7.357\end{array}$ & $\begin{array}{l}-2.230 \\
0.298\end{array}$ & $\begin{array}{l}-7.145 \\
1.814\end{array}$ & 0.134 & $\begin{array}{l}1.786 \\
5.335\end{array}$ & -0.275 & -352.4 & $\begin{array}{l}-2.781 \\
4.084\end{array}$ & $\begin{array}{l}-4.377 \\
2.474\end{array}$ & $\begin{array}{l}-2.610 \\
2.768\end{array}$ & -3.9321 .227 & -273.90 & $\begin{array}{l}-2.635 \\
8.008\end{array}$ \\
\hline Upper 95\% Cl & & & & & 0.461 & & 0.380 & 53.0 & & & & & 131.95 & \\
\hline - & - & - & - & - & - & - & - & - & - & - & - & - & - & - \\
\hline Number & 306 & 262 & 98 & 99 & 165 & 286 & 116 & 389 & 229 & 252 & 419 & 360 & 173 & 100 \\
\hline$P$ value & 0.197 & 0.902 & 0.134 & 0.243 & $<0.001$ & $<0.001$ & 0.753 & 0.148 & 0.710 & 0.586 & 0.954 & 0.304 & 0.493 & 0.322 \\
\hline I-square & $96 \%$ & $94 \%$ & $95 \%$ & $90 \%$ & $0.0 \%$ & $84 \%$ & $93 \%$ & $84 \%$ & $81 \%$ & $75 \%$ & $66 \%$ & $75 \%$ & $91 \%$ & $99 \%$ \\
\hline
\end{tabular}


Table 2 Results of main analysis and subgroup or sensitivity analyses (Continued)

\begin{tabular}{|c|c|c|c|c|c|c|c|c|c|c|c|c|c|c|}
\hline $\begin{array}{l}\text { Subgroup/ } \\
\text { Sensitivity }^{\mathrm{a}}\end{array}$ & $\begin{array}{l}\text { LVMi } \\
\mathrm{g} / \mathrm{m}^{2}\end{array}$ & $\begin{array}{l}\mathrm{EDVi} \\
\mathrm{ml} / \mathrm{m}^{2}\end{array}$ & $\begin{array}{l}\mathrm{IVS} \\
\mathrm{Mm}\end{array}$ & $\begin{array}{l}\text { VTD } \\
\mathrm{mm}\end{array}$ & $\begin{array}{l}\text { Cardiac index } \\
\mathrm{L} / \mathrm{min} / \mathrm{m}^{2}\end{array}$ & EF\% & $\begin{array}{l}\text { E/A } \\
\text { ratio }\end{array}$ & $\begin{array}{l}\text { NTpro-BNP } \\
\text { Pg/ml }\end{array}$ & $\begin{array}{l}\mathrm{HR} \\
\mathrm{bpm}\end{array}$ & $\begin{array}{l}\text { MAP } \\
\mathrm{mmHg}\end{array}$ & $\begin{array}{l}\text { SBP } \\
\mathrm{mmHg}\end{array}$ & $\begin{array}{l}\text { DBP } \\
\mathrm{mmHg}\end{array}$ & $\begin{array}{l}\text { SVRi dynes } \\
\mathrm{m}^{2} / \mathrm{sec} \mathrm{cm}^{-5}\end{array}$ & FMD\% \\
\hline $\begin{array}{l}\text { Right heart } \\
\text { disease }\end{array}$ & & & & & 0.309 & & & & -2.700 & & & 0.867 & & \\
\hline Lower 95\% Cl & / & / & / & / & 0.178 & / & / & / & -4.554 & / & / & -2.6374 .372 & / & / \\
\hline Upper 95\% Cl & & & & & 0.439 & & & & -0.847 & & & & & \\
\hline- & & & & & - & & & & - & & & - & & \\
\hline Number & & & & & 355 & & & & 347 & & & 30 & & \\
\hline$P$ value & / & / & / & / & $<0.001$ & / & / & / & 0.004 & / & / & 0.628 & / & / \\
\hline I-square & / & / & / & / & $0.0 \%$ & / & / & / & $0.0 \%$ & / & / & $0.0 \%$ & / & / \\
\hline $\begin{array}{l}\text { Non-cardiac } \\
\text { disease }\end{array}$ & & & & & & & & & -0.109 & & 0.107 & 0.073 & & 3.953 \\
\hline Lower 95\% Cl & / & / & / & / & / & I & / & / & -2.723 & / & -2.287 & -1.5151 .660 & / & 1.240 \\
\hline Upper 95\% Cl & & & & & & & & & 2.504 & & 2.502 & & & 6.667 \\
\hline-- & & & & & & & & & -- & & - - & - & & - - \\
\hline Number & & & & & & & & & 374 & & 374 & 374 & & 68 \\
\hline$P$ value & I & / & / & I & I & I & I & I & 0.935 & I & 0.930 & 0.928 & / & 0.004 \\
\hline I-square & I & I & 1 & I & I & 1 & 1 & I & $0.0 \%$ & I & $0.0 \%$ & $0.0 \%$ & I & $96 \%$ \\
\hline Age $<60$ yrs & & & & & 0.284 & 3.187 & $0.173^{a}$ & & & 1.940 & -0.008 & -0.292 & -130.44 & \\
\hline Lower 95\% Cl & & & & & 0.163 & -0.716 & -0.150 & & & -0.824 & -2.023 & -1.6311 .047 & -234.44 & / \\
\hline Upper $95 \% \mathrm{Cl}$ & / & / & / & / & 0.405 & 7.089 & 0.496 & / & / & 4.704 & 2.006 & - & -26.43 & \\
\hline-- & & & & & - & - & - & & & - & - & 513 & - & \\
\hline Number & & & & & 399 & 74 & 71 & & & 212 & 513 & & 89 & \\
\hline$P$ value & / & / & I & I & 0.000 & 0.110 & 0.295 & / & / & 0.196 & 0.993 & 0.669 & 0.014 & I \\
\hline I-square & 1 & I & I & 1 & $0.0 \%$ & $80 \%$ & $90 \%$ & 1 & 1 & $0.0 \%$ & $0.0 \%$ & $0.0 \%$ & $0.0 \%$ & I \\
\hline Age $\geq 60$ yrs & $-2.683^{\mathrm{a}}$ & 0.433 & & -2.666 & 0.354 & 2.582 & & $-40.199^{\mathbf{a}}$ & -0.491 & -2.349 & 0.663 & -2.048 & -57.48 & $3.558^{\mathrm{a}}$ \\
\hline Lower 95\% Cl & -9.169 & -6.49 & / & $\begin{array}{l}-7.145 \\
1.814\end{array}$ & 0.165 & -1.074 & / & -196.023 & -3.826 & -5.851 & -2.067 & -4.7410 .646 & -349.32 & 0.306 \\
\hline Upper 95\% Cl & 3.804 & 7.357 & & -- & 0.544 & 6.237 & & 115.624 & 2.844 & 1.153 & 3.392 & & 234.36 & 6.809 \\
\hline- & -- & - & & & - & - & & - & - & -- & - & - & - & - \\
\hline Nymber & 262 & 262 & & 99 & 121 & 167 & & 355 & 221 & 143 & 315 & 234 & 143 & 132 \\
\hline$P$ value & 0.418 & 0.902 & / & 0.243 & $<0.001$ & 0.166 & / & 0.613 & 0.773 & 0.189 & 0.634 & 0.136 & 0.699 & 0.032 \\
\hline I-square & $95 \%$ & $94 \%$ & / & $90 \%$ & $0.0 \%$ & $90 \%$ & / & $72 \%$ & $79 \%$ & $83 \%$ & $72 . \%$ & $71 \%$ & $94 \%$ & $99 \%$ \\
\hline
\end{tabular}


Table 2 Results of main analysis and subgroup or sensitivity analyses (Continued)

\begin{tabular}{|c|c|c|c|c|c|c|c|c|c|c|c|c|c|c|}
\hline $\begin{array}{l}\text { Subgroup/ } \\
\text { Sensitivity }^{\mathrm{a}}\end{array}$ & $\begin{array}{l}\mathrm{LVMi} \\
\mathrm{g} / \mathrm{m}^{2}\end{array}$ & $\begin{array}{l}\mathrm{EDVi} \\
\mathrm{ml} / \mathrm{m}^{2}\end{array}$ & $\begin{array}{l}\text { IVS } \\
\mathrm{Mm}\end{array}$ & $\begin{array}{l}\text { VTD } \\
\mathrm{mm}\end{array}$ & $\begin{array}{l}\text { Cardiac index } \\
\mathrm{L} / \mathrm{min} / \mathrm{m}^{2}\end{array}$ & $\mathrm{EF} \%$ & $\begin{array}{l}\mathrm{E} / \mathrm{A} \\
\text { ratio }\end{array}$ & $\begin{array}{l}\text { NTpro-BNP } \\
\mathrm{Pg} / \mathrm{ml}\end{array}$ & $\begin{array}{l}\text { HR } \\
\text { bpm }\end{array}$ & $\begin{array}{l}\text { MAP } \\
\mathrm{mmHg}\end{array}$ & $\begin{array}{l}\text { SBP } \\
\mathrm{mmHg}\end{array}$ & $\begin{array}{l}\mathrm{DBP} \\
\mathrm{mmHg}\end{array}$ & $\begin{array}{l}\text { SVRi dynes } \\
\mathrm{m}^{2} / \mathrm{sec} \mathrm{cm}^{-5}\end{array}$ & FMD\% \\
\hline Sildenafil & -4.022 & 0.433 & -0.966 & -2.666 & $0.289^{a}$ & 3.561 & 0.053 & -161.9 & -0.943 & $-0.880^{\mathrm{a}}$ & 0.248 & -0.995 & -70.9 & 2.7 \\
\hline Lower 95\% Cl & -10.137 & -6.49 & -2.230 & $\begin{array}{l}-7.145 \\
1.814\end{array}$ & 0.179 & 1.786 & -0.275 & -351.5 & -2.959 & -3.642 & -2.138 & -2.8520 .863 & -273.9 & -2.6 \\
\hline Upper 95\% Cl & 2.093 & 7.357 & 0.298 & & 0.399 & 5.335 & 0.380 & 27.6 & 1.073 & 1.882 & 2.634 & & 131.9 & 8.0 \\
\hline- & -- & - & -- & - & -- & -- & -- & -- & -- & -- & -- & -- & - & -- \\
\hline Number & 306 & 262 & 98 & 99 & 461 & 286 & 116 & 407 & 536 & 296 & 446 & 409 & 173 & 100 \\
\hline$P$ value & 0.197 & 0.902 & 0.134 & 0.243 & $<0.001$ & $<0.001$ & 0.753 & 0.094 & 0.359 & 0.532 & 0.839 & 0.294 & 0.493 & 0.322 \\
\hline I-square & $96 \%$ & $94 \%$ & $95 \%$ & $90 \%$ & $0.0 \%$ & $84 \%$ & $93 \%$ & $82 \%$ & $0.0 \%$ & $72 \%$ & $60 \%$ & $63 \%$ & $91 \%$ & / \\
\hline Tadalafil & & & & & & & & & -1.033 & & 4.659 & 1.101 & & 3.953 \\
\hline Lower 95\% Cl & & & & & & & & & -7.141 & & -2.529 & & / & 1.240 \\
\hline Upper 95\% Cl & / & / & I & / & / & / & / & / & 5.075 & / & 11.846 & -4.7176 .920 & & 6.667 \\
\hline- & & & & & & & & & - & & -- & - & & -- \\
\hline Number & & & & & & & & & 32 & & 32 & 32 & & 68 \\
\hline$P$ value & / & / & / & / & / & / & / & / & 0.740 & / & 0.204 & 0.711 & / & 0.004 \\
\hline I-square & I & I & / & / & / & / & I & I & $0.0 \%$ & / & $0.0 \%$ & $0.0 \%$ & I & I \\
\hline Vardenafil & & & & & & & & & -0.408 & & & & & \\
\hline Lower 95\% Cl & & & & & & & & & -3.746 & & & & & \\
\hline Upper 95\% Cl & / & / & / & / & / & / & / & / & 2.931 & / & / & / & / & / \\
\hline- & & & & & & & & & - - & & & & & \\
\hline Number & & & & & & & & & 409 & & & & & \\
\hline$P$ value & / & / & / & / & / & / & / & / & 0.811 & / & / & / & / & / \\
\hline I-square & I & I & I & I & / & I & / & / & $0.0 \%$ & / & / & / & / & / \\
\hline
\end{tabular}




\section{Cardiac performance}

Cardiac index and EF were considered measures of systolic performance and E/A ratio a measure of diastolic function.

Data on the cardiac index were available in seven studies $[2,10,11,25,30,32,40]$ (520 patients, $65.6 \%$ male), showing an increase of $0.304 \mathrm{~L} / \mathrm{min} / \mathrm{m}^{2}$ (95\% CI: $0.202 ; 0.406$, $P<0.001)$ induced by sildenafil $[2,10,11,25,32,40]$ and vardenafil [30] over placebo [32] (Figure 3). Subgroup analyses confirmed the significant effects on the cardiac index in patients without LVH $\left(0.354 \mathrm{~L} / \mathrm{min} / \mathrm{m}^{2} ; 95 \% \mathrm{CI}\right.$ : $0.165 ; 0.544, P<0.001)$ and in patients with left $(0.298$ $\mathrm{L} / \mathrm{min} / \mathrm{m}^{2} ; 95 \%$ CI: $\left.0.134 ; 0.461, P<0.001\right)$ or right heart disease $\left(0.309 \mathrm{~L} / \mathrm{min} / \mathrm{m}^{2}\right.$; $95 \%$ CI: $\left.0.178 ; 0.438, P<0.001\right)$. The PDE5i-dependent increase in the cardiac index was observed in patients both younger and older than the age of 60 (Table 2). These results were confirmed when sensitivity analysis was performed and the study using vardenafil [30] excluded $\left(0.289 \mathrm{~L} / \mathrm{min} / \mathrm{m}^{2} ; 95 \%\right.$ CI: 0.179 ; 0.399, $P<0.001$ ).

Six studies [10-12,24,25,31] (286 patients, 95.8\% male) gave details on EF in patients with left heart disease, in whom sildenafil $(\mathrm{n}=146)$ produced a $3.561 \%(95 \%$ CI: 1.786 ; 5.335$)$ increase over placebo $(\mathrm{n}=140, P<0.001)$ (Figure 3). Subgroup analyses revealed larger effect sizes in patients with LVH (4.382\%; 95\% CI: 2.059; 6.705, $P<0.001)$. The meta-analysis of $\mathrm{E} / \mathrm{A}$ ratio included three studies [12,25,37] (116 patients, 83.7\% male) and showed no significant effect of sildenafil over placebo.

\section{Neuroendocrine biomarkers}

NT-proBNP did not change $(-161.9 \mathrm{pg} / \mathrm{mL} ; 95 \%$ CI: -351.5 ; 27.6, $P=0.10)$ in six trials $[10-14,40](\mathrm{n}=407$; $76 \%$ male, all treated with sildenafil or placebo). Subgroup analysis showed a significant decrease in NT-proBNP levels in patients with LVH $(486.7 \mathrm{pg} / \mathrm{mL}$; 95\% CI: -712.8 ; $-260.7, P<0.001$ ) (Figure 4).

\section{Hemodynamic parameters}

PDE5i administration had no effect on HR in the main analysis ( $\mathrm{n}=977,71 \%$ male) $[2,10,11,20,24,26,28,30,31,35$, 37,39] (Figure 5). In the subgroup analysis a significant decrease in HR was found in patients with right heart disease (2.7 bpm; CI: $-4.5 ;-0.8, P=0.004)$.

In the 16 analyzed studies $(\mathrm{n}=1,012)$, systolic and diastolic BP did not change (SBP; $+0.616 \mathrm{mmHg} ; 95 \%$ CI: -1.113 ; $2.345, P=0.49$; DBP: $-0.763 \mathrm{mmHg}$; $95 \%$ CI: -2.174 ; $0.648, P=0.30$; MAP: $-0.413 \mathrm{mmHg}$; $95 \%$ CI: $-3.023 ; 2.196, P=0.80) \quad[10-12,14,20,23-26,28,30$, $31,36,38,39$ ] (see Figure 2 and Additional file 1: Figure S1). Similarly, no change was found in SVRi $[10,25,30,31,39]$ $(\mathrm{n}=232,71 \%$ male) [see Additional file 1: Figure S1]. Subgroup analysis showed a significant decrease in SVRi only in patients younger than $60\left(-130.44\right.$ dynes $\mathrm{m}^{2} / \mathrm{sec} \mathrm{cm}^{-5}$; 95\% CI: $-234.44 ;-26.43, P=0.010)$.

\section{Endothelial function}

Four studies $[11,24,33,34](\mathrm{n}=168$, all males) showed an increase in FMD (3.306\%; 95\% CI: 0.530; 6.082, $P=0.020$ ) after PDE5i administration over placebo [see Additional file 1: Figure S2]. Subgroup analysis revealed a higher effect size in FMD of patients without cardiac disease treated with tadalafil (3.953\%; 95\% CI: 1.24; 6.667, $P=$ 0.004). Sensitivity analysis showed a larger effect size in FMD in patients older than $60(3.558 \%$; $95 \% \mathrm{CI}$ : $0.306 ; 6.809, P=0.030$ ).

\section{Safety data: PDE-5 inhibitors versus placebo \\ Adverse events}

In our analysis, the adverse events with the highest relative risks (RRs) related to PDE5i treatment were flushing or rash $(\mathrm{RR}=3.406$; $95 \% \mathrm{CI}$ : 1.628 ; $7.126, P=0.001)$, headache $(\mathrm{RR}=2.507 ; 95 \% \mathrm{CI}: 1.416 ; 4.439, P=0.002)$, gastric symptoms $(\mathrm{RR}=4.138 ; 95 \% \mathrm{CI}: 1.564 ; 10.946, P=0.004)$ and epistaxis $(\mathrm{RR}=4.701 ; 95 \% \mathrm{CI}: 1.314 ; 16.812, P=0.017)$ [see Additional file 1: Table S3]. No significant risk was observed in other reported side effects including: intestinal and musculoskeletal symptoms, nasopharyngitis, dizziness or tinnitus, visual disturbance and photosensitivity, skin irritation, insomnia, pruritus and dyspnea. The incidence of symptomatic hypotension and other serious adverse events was not statistically different between PDE5i and placebo treated groups.

\section{Discussion}

This meta-analysis suggests for the first time that longterm daily PDE5i administration in mixed study populations: (1) exerts an anti-remodeling effect on cardiac geometry in patients with moderate-severe LVH, and (2) improves cardiac performance in all subjects with different clinical settings, with (3) no major changes in hemodynamic parameters and (4) a good safety profile. This analysis clarifies the discrepancies in previous trials and suggests that PDE5i is efficacious in cardiac protection in different stages of heart disease.

\section{Effect of PDE5i on cardiac geometry}

The main analysis performed on all studies found no consistent changes in cardiac geometry. In the few studies performed on patients with severe LVH, PDE5i decreased LVMi $\left(-12.207 \mathrm{~g} / \mathrm{m}^{2}\right.$; CI: -18.846 to $\left.-5.568, P<0.001\right)$, with the limitation being the small number of treated subjects. Nevertheless, the size of this reduction seems to be clinically relevant if compared to other antiremodeling drugs such as ACEi (angiotensin converting enzyme inhibitors), ARBs (angiotensin II receptor blockers) or spironolactone, that have been shown to decrease LVMi, 


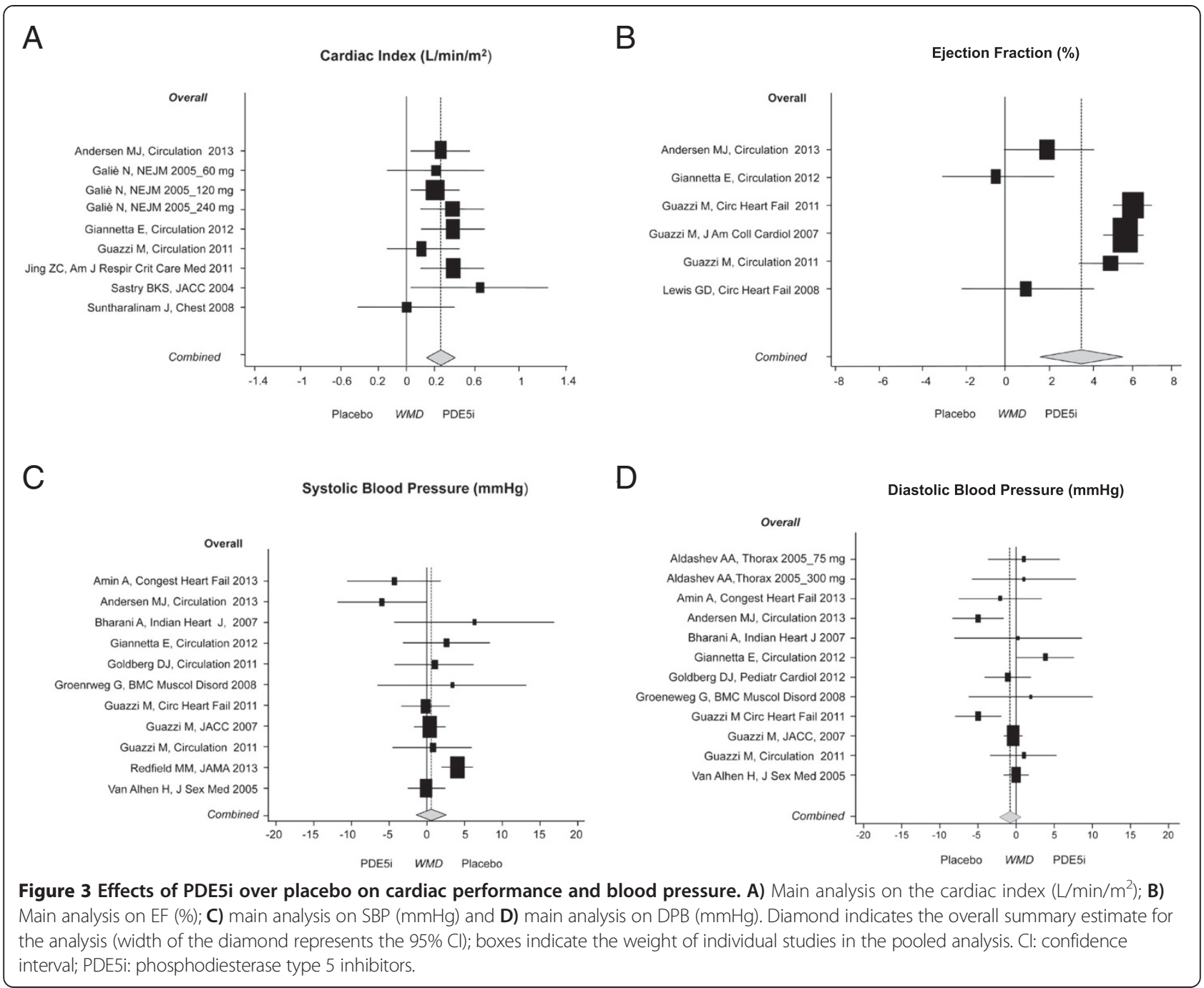

respectively, by $11.97 \mathrm{~g} / \mathrm{m}^{2}$ [41], $16.68 \mathrm{~g} / \mathrm{m}^{2}$ [41] and $11 \mathrm{~g} / \mathrm{m}^{2}$ in patients with congestive HF [42]. Therefore, as observed in animal studies [9], our meta-analysis shows that in human LVH, chronic sildenafil improves cardiac geometry to an extent comparable to that of currently recommended anti-remodeling therapies, whereas in patients with normal or near-normal LVMi, PDE5i produced no measurable change in cardiac mass.

Of note is the high heterogeneity emerging from the main analysis $\left(\mathrm{I}^{2}=96 \%, P<0.001\right)$, that improved when the studies were stratified according to the degree of cardiac hypertrophy at baseline (LVH: $\mathrm{I}^{2}=88 \%, P=0.003$; non $/$ mild LVH: $\left.\mathrm{I}^{2}=80 \%, P=0.005\right)$. In addition, inconsistencies in the response to treatment seem related to the study duration. In the two trials lasting less than six months $[10,11]$, LVMi apparently increased $\left(+3.011 \mathrm{~g} / \mathrm{m}^{2}\right.$; CI -0.506 to $5.515 ; P=0.018$ ). Specifically, Andersen et al. enrolled subjects immediately after an acute myocardial infarction and the within-subject analysis showed no significant LVMi changes in either sildenafil (baseline:
$93 \pm 19 \mathrm{~g} / \mathrm{m}^{2}$, post-treatment: $95 \pm 20 \mathrm{~g} / \mathrm{m}^{2}$ ) or placebo (baseline $93 \pm 20 \mathrm{~g} / \mathrm{m}^{2}$, post-treatment $91 \pm 18 \mathrm{~g} / \mathrm{m}^{2}$ ) treated subjects. However, given the opposite trends, the calculated WMD returned a positive effect. PDE5i have been shown to exert cardio-protection against postischemic fibrosis [6] and the post-acute setting of this study could account for the observed WMD (Figure 2). In contrast, Giannetta et al. enrolled type 2 diabetic patients with an increased LVMi $\left(119.4 \pm 25.7 \mathrm{~g} / \mathrm{m}^{2}\right)$, that did not change after sildenafil or placebo (respectively, $-0.67 \pm 5.07 \mathrm{~g} / \mathrm{m}^{2}$ and $-2.03 \pm 7.64 \mathrm{~g} / \mathrm{m}^{2}$ ). For all these reasons, the observed LVMi variation in this subgroup of studies could not be considered clinically relevant.

In the three studies lasting more than six months $[12,14,25]$, LVMi decreased to a much greater extent $\left(-8.446 \mathrm{~g} / \mathrm{m}^{2} ; 95 \% \mathrm{CI}-15.694\right.$ to $\left.-1.197 ; P=0.022\right)$. The two studies by Guazzi et al. were performed in different populations with severe $\mathrm{LVH}$ due to $\mathrm{HF}$ of various etiologies (baseline LVMi $=166.4 \pm 12.1 \mathrm{~g} / \mathrm{m}^{2}[25]$ and $\left.147.2 \pm 30.2 \mathrm{~g} / \mathrm{m}^{2}[12]\right)$ and were analyzed along with 


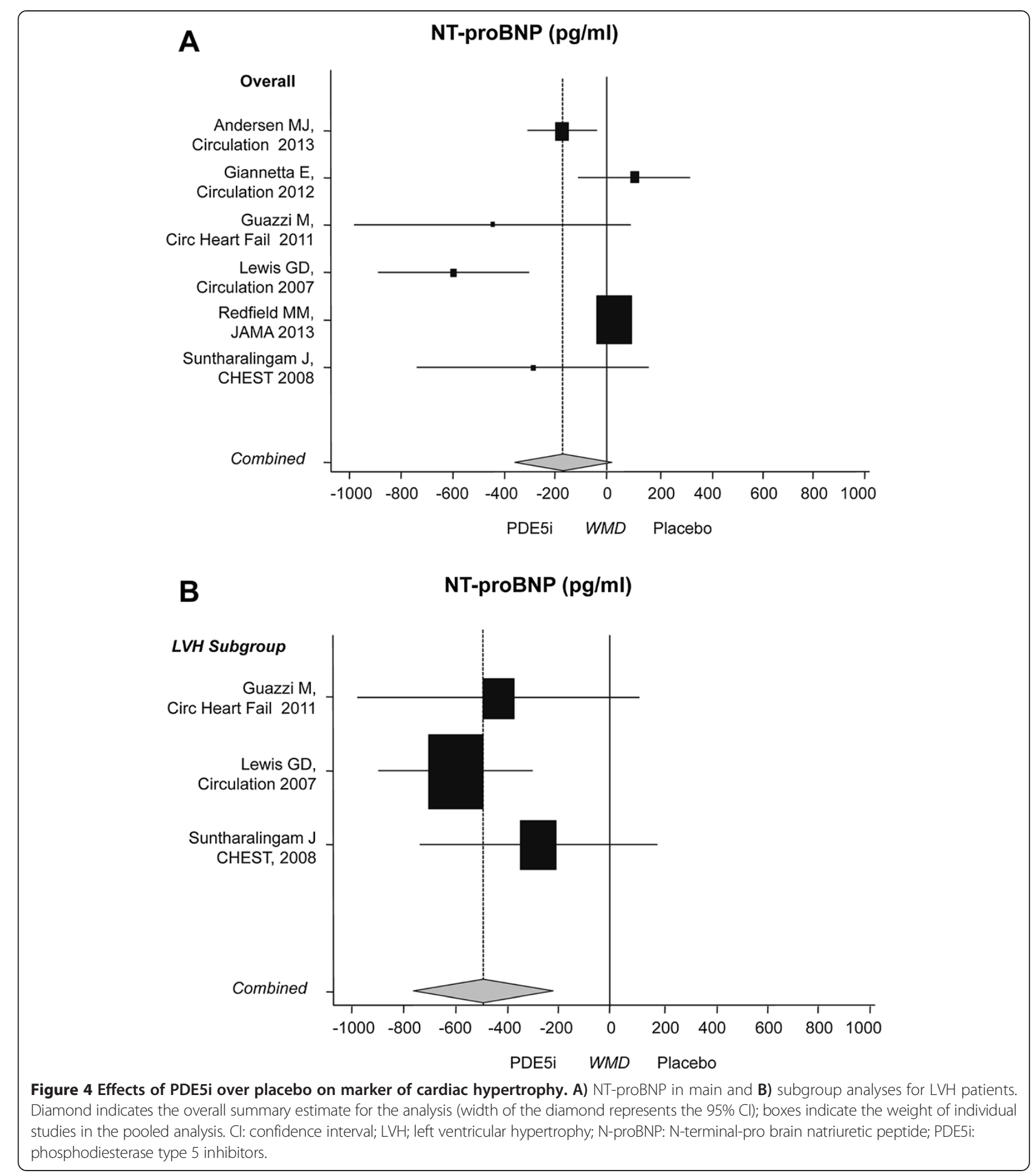

the study by Redfield et al. performed in non-LVH subjects (baseline LVMi $=65 \mathrm{~g} / \mathrm{m}^{2}$; interquartile range (IQR): 54 to 78) [14]. Based on these findings, it is reasonable to assume that a certain duration of treatment and a degree of hypertrophy are necessary to observe a change in the cardiac mass as compared to changes in other geometric
(EDV) and functional (EF) parameters, that might occur earlier. Length of treatment and degree of hypertrophy offer a clinical explanation to the apparently conflicting findings of the main analysis.

Cardiac remodeling is also measured with the volume-tomass ratio known as the concentricity index (LVM/EDV) 


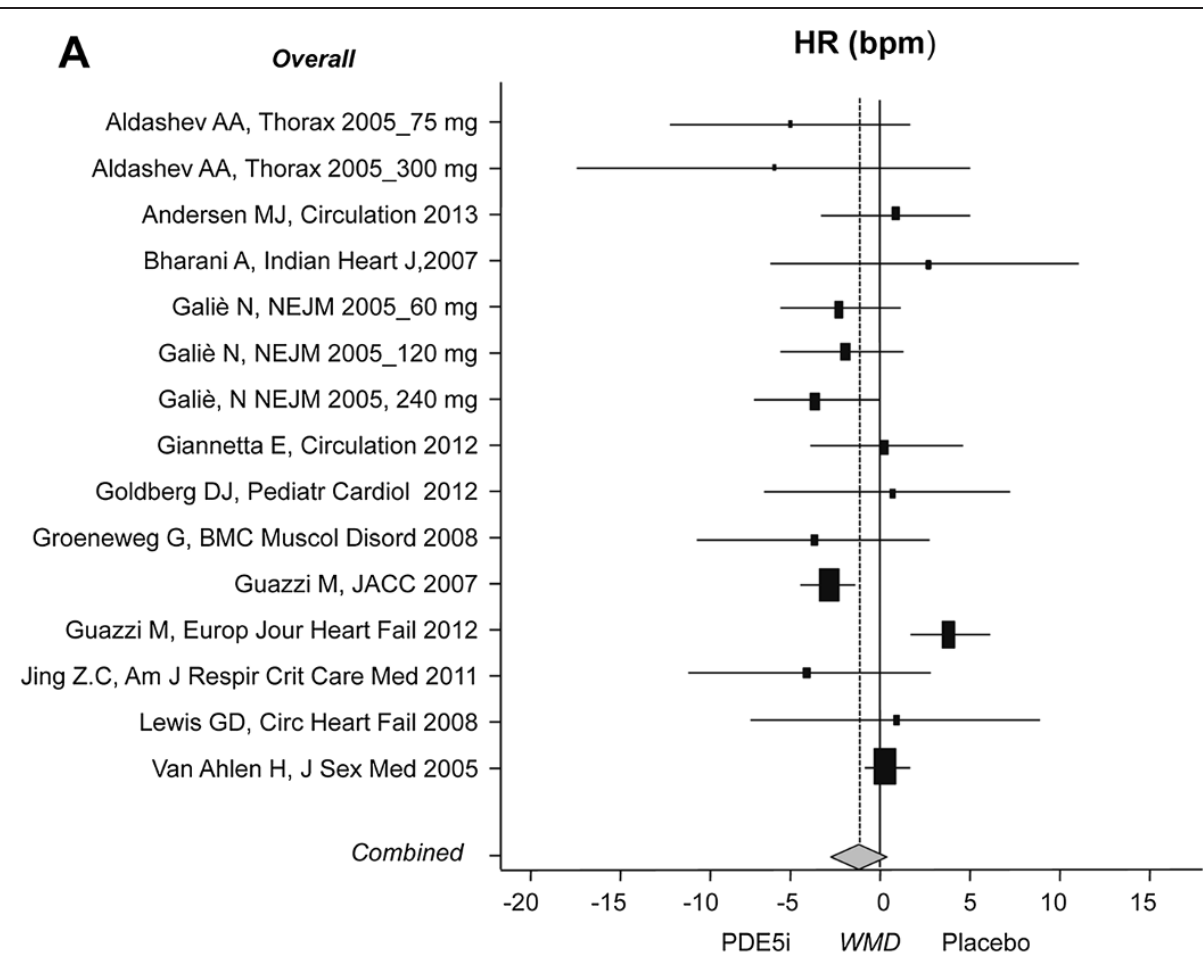

B

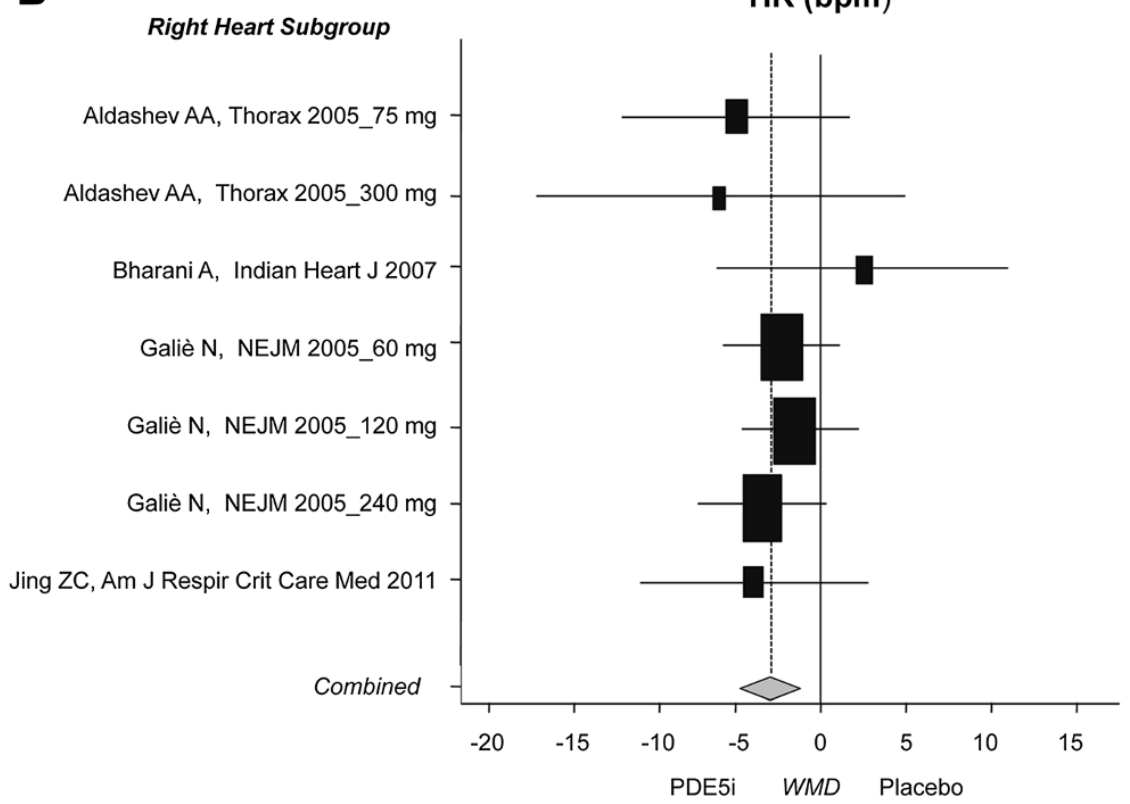

Figure 5 Effects of PDE5i on heart rate. A) Main analysis on heart rate (HR); B) subgroup analysis on patients with right heart disease. Diamond indicates the overall summary estimate for the analysis (width of the diamond represents the $95 \% \mathrm{Cl}$ ); boxes indicate the weight of individual studies in the pooled analysis. Cl: confidence interval; PDE5i: phosphodiesterase type 5 inhibitors.

[43]. The main analysis of EDVi revealed high heterogeneity (I2 =94\%; $P<0.001$ ), but when restricting the analysis to non/mild LVH it dropped to $0 \%(P=0.53)$. The apparently contradictory findings are probably explained by the significant differences in the characteristics of the enrolled study populations. The study by Guazzi et al. was the only study performed in subjects with ventricle dilatation and reduced EF (severe LVH heart failure); in fact, in the sensitivity analysis performed in non/mild-LVH patients, we detected a significant increase in EDVi $\left(4.999 \mathrm{~mL} / \mathrm{m}^{2} ; 95 \%\right.$ 
CI: $3.286 ; 6.711, P<0.001, \mathrm{I}^{2}$ of $\left.0 \%\right)$. Since EDVi may increase in the final stages of heart failure (eccentric hypertrophy), but may also drop in the earlier stages of mild hypertrophy (concentric hypertrophy), we believe that it is clinically meaningful to attribute the observed EDVi change in the three homogeneous studies to improved relaxation of the ventricular wall. Notably, the LVM/EDV ratio in all of these trials was consistent with a 'concentric thick LVH' pattern $[10,11,14]$, suggesting that at this earlier stage PDE5i improves myocardial wall viscoelastic properties resulting in a better diastolic load.

\section{Effect of PDE5i on cardiac performance}

Our main analysis reveals for the first time a statistically and clinically meaningful improvement in cardiac performance, cardiac index and EF.

In our main analysis, the cardiac index improves under PDE5i with no heterogeneity between the studies $\left(\mathrm{I}^{2}=0.0 \%\right)$, despite the differences in the baseline characteristics of the sample analyzed.

After dividing the sample according to the cardiovascular features, the subgroup analyses confirmed that PDE5i increases the cardiac index by $0.3 \mathrm{~L} / \mathrm{min} / \mathrm{m}^{2}$ in non-LVH patients. In $\mathrm{LVH}$ patients the increase in the cardiac index does not reach statistical significance. However, this result is probably due to the paucity of available data, with only two studies $[25,40]$ involving a total of 62 subjects, one of which was performed in men with chronic thromboembolic pulmonary hypertension.

In the main analysis, EF also improves, but with highly significant heterogeneity ( $\left.\mathrm{I}^{2} 84 \%\right)$. Pooling studies according to $\mathrm{LVH}$, we observed that in subjects with $\mathrm{LVH}$ the improvement in EF was greater and with lower heterogeneity (4.38\%, CI: 2.059; 6.705, $P<0.001 ; \mathrm{I}^{2}=79 \%$ ), comparable to the average change obtained in large trials using the currently recommended therapies for HF. A recent meta-analysis [44] comparing different beta blockers in $\mathrm{HF}$ with reduced EF reported a WMD for EF of $4.1 \%$ (95\% CI: 3.1; 4.9) versus placebo/standard treatment. Conversely, in non-hypertrophic subjects with preserved EF the change was not statistically significant $(0.973 \%$, CI: -1.329 ; 3.274, $P<0.408 ; \mathrm{I}^{2}=0.0 \%$ ). These results are consistent with the fact that the PDE5 enzyme is expressed at low levels within cardiomyocytes, until LVH hypertrophy and failure develop [45]. Our data show that only when the compensatory mechanisms evolve into changes in cardiac geometry $[14,25]$ do the benefits of PDE5i on EF become clinically detectable.

A possible explanation for the observed heterogeneity is that PDE5 inhibitors work only when the target enzyme is overexpressed, a condition that parallels the degree of hypertrophy [see Additional file 1-Limitations]. In addition, the cardiac index, a more stable measure of cardiac performance, being a function of both chambers' volumes and contractility, showed no significant heterogeneity for both the main and subgroup analyses. A recent analysis reported PDE5i-induced changes in EF in HF patients [46]. However, it omitted some relevant studies [25,31] and misclassified one trial [25], undermining the relevance of the findings. This led to incomplete data and methodological flaws when the authors attempted to perform a sub-group analysis within a group of just two studies. As EF and the cardiac index depend on cardiac contractility, hemodynamics and $H R$, the fact that we found no significant changes in hemodynamics and HR suggests that the improvement in cardiac performance observed in patients with left heart disease $[10,11,25]$ is the result of a PDE5i-mediated effect on cardiac contractility, while in patients with right heart disease a dual mechanism of better pulmonary hemodynamics/oxygenation and improved inotropy is likely to occur.

One novelty of our work is its parallel analysis, for the first time, of the changes induced in cardiac geometry and performance, allowing an appraisal of the direct myocardial action of PDE5i in humans; until now, this had been considered merely secondary to indirect systemic/ pulmonary hemodynamic changes.

\section{Neuroendocrine biomarkers}

Analyses on NT-proBNP level, an indicator of myocardial stretch [47], follow the trend observed for ventricular mass and diastolic function [48,49], with a significant improvement in the subgroup of hypertrophic patients.

\section{Effect of PDE5i on hemodynamic parameters and endothelial function}

No statistically significant chronotropic effect was found for PDE5i in the main analysis, except for the subgroup of patients with right heart disease [see Additional file 1: Figure S1], where PDE5i reduced HR, without heterogeneity (see Table 2 and Additional file 1-Heterogeneity). For this group, the finding could be explained in terms of better oxygenation. The chronotropic effects of PDE5i have never been investigated, but a previous human study shows that sildenafil can blunt beta-adrenergic stimulation [50] and the findings are also consistent with a general improvement in cardiac contractility [51]. A reduction of just $5 \mathrm{bpm}$ in patients with $\mathrm{HF}$ has been associated with a lower risk of cardiovascular death and hospitalization [51], but drugs with a proven negative chronotropic effect, such as beta-blockers, produce HR reductions in the 8- to15bpm range [51].

Regarding the effects of PDE5i on BP, our analysis shows that PDE5i given in a continuous regimen have no sustained effects on systemic BP, in a large group of disorders. This effect is confirmed by the absence of any relevant effect of PDE5i on SVRi in the main analysis. Our results confirm the findings described in men with 
anatomically severe coronary disease [52] where the hypothesis of a coronary steal syndrome due to PDE5irelated vasodilation was excluded using cardiac catheterism, and are consistent with those of a smaller meta-analysis [53].

Analysis of the little data available on endothelial function $[11,12,34]$ seems to confirm a beneficial effect of continuous treatment with PDE5i, although the data were almost entirely derived from ED patients [54,55], the effects did not reach statistical significance in patients with left heart disease, and there was very high heterogeneity $\left(\mathrm{I}^{2}=99 \%, P<0.001\right)$. Only the smallest [33] of the four trials analyzed is within the $95 \%$ CIs, and no relevant improvement was obtained by subgroup or sensitivity analysis [see Additional file 1-Heterogeneity], thus limiting the validity of the findings.

\section{Adverse events}

Our analysis of the side effects of long-term daily use of PDE5i showed that only flushing, headache, epistaxis and gastric symptoms are statistically related to these drugs, confirming their cardiovascular safety and good tolerability. This is relevant in case future studies are planned to test PDE5i for cardiovascular disorders. It is worth noting that the majority of retrieved studies were performed in aging subjects, supporting the safety profile of these drugs when administered as a daily or continuous regimen even in this age group.

\section{Limitations}

Our review has some limitations. The first concerns the differences in the baseline characteristics. Subgroup analyses for length of treatment (less than or more than six months) were not possible due to the small number of studies. Similarly, the majority of eligible trials provided data obtained in patients older than 60 years, using sildenafil. Furthermore, the lack of data on gender differences in cardiac outcomes did not permit the exploration of male and female behavior, whereas there have been significant advances with respect to cardiovascular disease in women in the last decade [56].

The second limitation pertains to the often missing information on cardiac geometry parameters; while considering that for all outcomes, $25 \%$ of trials had a risk of reporting bias.

The third concern is that the findings of this study are nonetheless prone to publication bias and that 13 out of 24 trials had unclear potential for selection bias (although none with a high risk), that may threaten their validity. On the positive side, industrial bias seems limited, as most trials were spontaneous studies that received only external support. The fact that some studies did not report data on all basic outcomes reinforces the value of the COMET initiative aimed at defining the minimum standardized set of outcomes that each clinical trial should report for specific health conditions [57].

A fourth concern is on the high heterogeneity found in the main analysis. In most, but not all, cases, this was reasonably explained by the inclusion criteria of the trials, degree of hypertrophy and chamber dilation, or by duration of treatment.

Finally, conclusions drawn from subgroup analyses considering only two studies are only indicative and should be interpreted with care. See specific limitations in Additional file 1-Limitations.

\section{Conclusions}

With respect to the initial purpose of the study, we showed that in selected cohorts long-term continuous PDE5i administration can produce clinically meaningful improvements in cardiac remodeling and performance with an excellent cardiovascular safety and tolerability profile even in older patients and under prolonged use. The findings are based on recent RCTs that, albeit wellperformed, involved small and heterogeneous populations. With these limitations, our analyses suggest that in humans, in vivo, PDE5i exert anti-remodeling properties that become evident only when the hypertrophic trigger results in overexpression of the targeted enzyme in cardiomyocytes. The effects are initially seen as an improvement in diastolic function (LVM/EDV ratio) and, subsequently, as a reduction in LVMi in patients with moderate to severe LVH. All the above findings occur without any relevant changes in hemodynamics, as evaluated through systemic BP and SVRi.

Our analyses reveal that the ideal target population to benefit from PDE5 is patients with HF and LVH. Given the paucity of published data, on the basis of these encouraging findings large clinical trials are urgently needed on the long-term effects of continuous PDE5i administration, focusing on cardiovascular outcomes and sexspecific response in these patients.

\section{Additional file}

Additional file 1: Additional statistical analyses, heterogeneity, risk of bias, limitations, additional tables and figures.

\section{Abbreviations}

ACEi: angiotensin converting enzyme inhibitors; ARBs: angiotensin II receptor blockers; BP: blood pressure; bpm: beats per minute; CAMP: cyclic adenosine monophosphate; cGMP: cyclic guanosine monophosphate; Cl: confidence interval; DB: double-blind; DBP: diastolic blood pressure; E/A ratio: the ratio of the early $(E)$ to late $(A)$ ventricular filling velocities; $E D$ : erectile dysfunction; EDVi: end-diastolic volume index; EF: ejection fraction; FMD: flow mediated vasodilation; HF: heart failure; HR: heart rate; IQR: interquartile range; IVS: interventricular septum; LVH: left ventricular hypertrophy; LVMi: left ventricular mass index; MAP: mean arterial blood pressure; NTpro-BNP: $\mathrm{N}$-terminal-pro brain natriuretic peptide; $\mathrm{PAH}$ : pulmonary arterial hypertension; PC: placebo-controlled; PDE1: phosphodiesterase type 1; 
PDE2: phosphodiesterase type 2; PDE5i: phosphodiesterase type 5 inhibitors; RCTs: randomikzed controlled trials; RR: relative risk; SBP: systolic blood pressure; SD: standard deviation; SVRi: systemic vascular resistance index; VTD: ventricular transverse diameter; Wks: weeks; WMD: weighted mean difference.

\section{Competing interests}

The authors declare that they have no competing interests.

\section{Authors' contributions}

$E G, D G, T F, R P$ and $A M I$ contributed to the study conception and design and the acquisition and interpretation of data. VDA, TF, RP and EG performed the analysis. All authors were involved in drafting and critical revision for important intellectual content. All authors read and approved the final manuscript.

\section{Acknowledgements}

The authors thank Marie-Hélène Hayles for revision of the English text and the staff at the Interdepartmental Experimental and Molecular Medicine Library (bmedsper) of Sapienza University of Rome for their help in the bibliographic search.

\section{Sources of funding}

The study was supported by Ministry of Research MIUR Grant FIRB no. RBAP109BLT.

\section{Author details}

'Department of Experimental Medicine, Sapienza University of Rome, Viale del Policlinico 155, Rome 00161, Italy. ${ }^{2}$ Unit of Clinical and Molecular Epidemiology, IRCCS San Raffaele Pisana of Rome, Via della Pisana 235, Rome 00163, Italy. ${ }^{3}$ Department of Cardiovascular and Respiratory Science, Sapienza University of Rome, Piazzale Aldo Moro 5, Rome 00185, Italy.

Received: 11 July 2014 Accepted: 17 September 2014

Published online: 20 October 2014

\section{References}

1. Tsertsvadze A, Fink HA, Yazdi F, MacDonald R, Bella AJ, Ansari MT, Garritty C, Soares-Weiser K, Daniel R, Sampson M, Fox S, Moher D, Wilt TJ: Oral phosphodiesterase- 5 inhibitors and hormonal treatments for erectile dysfunction: a systematic review and meta-analysis. Ann Intern Med 2009, 151:650-661.

2. Galie N, Ghofrani HA, Torbicki A, Barst RJ, Rubin LJ, Badesch D, Fleming T, Parpia T, Burgess G, Branzi A, Grimminger F, Kurzyna M, Simonneau G: Sildenafil citrate therapy for pulmonary arterial hypertension. N Engl J Med 2005, 353:2148-2157.

3. Michelakis ED, Tymchak W, Noga M, Webster L, Wu XC, Lien D, Wang SH, Modry D, Archer SL: Long-term treatment with oral sildenafil is safe and improves functional capacity and hemodynamics in patients with pulmonary arterial hypertension. Circulation 2003, 108:2066-2069.

4. Napoli C, Loscalzo J: Nitric oxide and other novel therapies for pulmonary hypertension. J Cardiovasc Pharmacol Ther 2004, 9:1-8.

5. Egerdie RB, Auerbach S, Roehrborn CG, Costa P, Garza MS, Esler AL, Wong $D G$, Secrest RJ: Tadalafil 2.5 or $5 \mathrm{mg}$ administered once daily for 12 weeks in men with both erectile dysfunction and signs and symptoms of benign prostatic hyperplasia: results of a randomized, placebo-controlled, double-blind study. J Sex Med 2012, 9:271-281.

6. Kukreja RC: Cardiovascular protection with sildenafil following chronic inhibition of nitric oxide synthase. Br J Pharmacol 2007, 150:538-540

7. Opie LH, Commerford PJ, Gersh BJ, Pfeffer MA: Controversies in ventricular remodelling. Lancet 2006, 367:356-367.

8. Kass DA: Cardiac role of cyclic-GMP hydrolyzing phosphodiesterase type 5: from experimental models to clinical trials. Curr Heart Fail Rep 2012, 9:192-199.

9. Takimoto E, Koitabashi N, Hsu S, Ketner EA, Zhang M, Nagayama T, Bedja D, Gabrielson KL, Blanton R, Siderovski DP, Mendelsohn ME, Kass DA: Regulator of $\mathrm{G}$ protein signaling 2 mediates cardiac compensation to pressure overload and antihypertrophic effects of PDE5 inhibition in mice. J Clin Invest 2009, 119:408-420.

10. Andersen MJ, Ersboll M, Axelsson A, Gustafsson F, Hassager C, Kober L, Borlaug BA, Boesgaard S, Skovgaard LT, Moller JE: Sildenafil and diastolic dysfunction after acute myocardial infarction in patients with preserved ejection fraction: the Sildenafil and Diastolic Dysfunction After Acute Myocardial Infarction (SIDAMI) trial. Circulation 2013, 127:1200-1208.

11. Giannetta E, Isidori AM, Galea N, Carbone I, Mandosi E, Vizza CD, Naro F, Morano S, Fedele F, Lenzi A: Chronic inhibition of cGMP phosphodiesterase $5 \mathrm{~A}$ improves diabetic cardiomyopathy: a randomized controlled clinical trial using magnetic resonance imaging with myocardial tagging. Circulation 2012, 125:2323-2333.

12. Guazzi M, Vicenzi M, Arena R, Guazzi MD: PDE5 inhibition with sildenafil improves left ventricular diastolic function, cardiac geometry, and clinical status in patients with stable systolic heart failure: results of a 1-year, prospective, randomized, placebo-controlled study. Circ Heart Fail 2011, 4:8-17.

13. Lewis GD, Shah R, Shahzad K, Camuso JM, Pappagianopoulos PP, Hung J, Tawakol A, Gerszten RE, Systrom DM, Bloch KD, Semigran MJ: Sildenafil improves exercise capacity and quality of life in patients with systolic heart failure and secondary pulmonary hypertension. Circulation 2007, 116:1555-1562.

14. Redfield MM, Chen HH, Borlaug BA, Semigran MJ, Lee KL, Lewis G, LeWinter MM, Rouleau JL, Bull DA, Mann DL, Deswal A, Stevenson LW, Givertz MM, Ofili EO, O'Connor CM, Felker GM, Goldsmith SR, Bart BA, McNulty SE, Ibarra JC, Lin G, Oh JK, Patel MR, Kim RJ, Tracy RP, Velazquez EJ, Anstrom KJ, Hernandez AF, Mascette AM, Braunwald E: Effect of phosphodiesterase-5 inhibition on exercise capacity and clinical status in heart failure with preserved ejection fraction: a randomized clinical trial. JAMA 2013, 309:1268-1277.

15. Ishikura F, Beppu S, Hamada T, Khandheria BK, Seward JB, Nehra A: Effects of sildenafil citrate (Viagra) combined with nitrate on the heart. Circulation 2000, 102:2516-2521.

16. Lindman BR, Zajarias A, Madrazo JA, Shah J, Gage BF, Novak E, Johnson SN, Chakinala MM, Hohn TA, Saghir M, Mann DL: Effects of phosphodiesterase type 5 inhibition on systemic and pulmonary hemodynamics and ventricular function in patients with severe symptomatic aortic stenosis. Circulation 2012, 125:2353-2362.

17. Katz SD, Balidemaj K, Homma S, Wu H, Wang J, Maybaum S: Acute type 5 phosphodiesterase inhibition with sildenafil enhances flow-mediated vasodilation in patients with chronic heart failure. J Am Coll Cardiol 2000 36:845-851.

18. Moher D, Liberati A, Tetzlaff J, Altman DG: Preferred reporting items for systematic reviews and meta-analyses: the PRISMA statement. I Clin Epidemiol 2009, 62:1006-1012.

19. Higgins JP, Altman DG, Gotzsche PC, Juni P, Moher D, Oxman AD, Savovic J, Schulz KF, Weeks L, Sterne JA: The Cochrane Collaboration's tool for assessing risk of bias in randomised trials. BMJ 2011, 343:d5928.

20. Groeneweg G, Huygen FJ, Niehof SP, Wesseldijk F, Bussmann JB, Schasfoort FC, Stronks DL, Zijlstra FJ: Effect of tadalafil on blood flow, pain, and function in chronic cold complex regional pain syndrome: a randomized controlled trial. BMC Musculoskelet Disord 2008, 9:143.

21. Lang RM, Bierig M, Devereux RB, Flachskampf FA, Foster E, Pellikka PA, Picard MH, Roman MJ, Seward J, Shanewise JS, Solomon SD, Spencer KT, Sutton MS, Stewart WJ: Recommendations for chamber quantification: a report from the American Society of Echocardiography's Guidelines and Standards Committee and the Chamber Quantification Writing Group, developed in conjunction with the European Association of Echocardiography, a branch of the European Society of Cardiology. J Am Soc Echocardiogr 2005, 18:1440-1463.

22. Januzzi JL Jr: The role of natriuretic peptide testing in guiding chronic heart failure management: review of available data and recommendations for use. Arch Cardiovasc Dis 2012, 105:40-50.

23. Behling A, Rohde LE, Colombo FC, Goldraich LA, Stein R, Clausell N: Effects of 5'-phosphodiesterase four-week long inhibition with sildenafil in patients with chronic heart failure: a double-blind, placebo-controlled clinical trial. J Card Fail 2008, 14:189-197.

24. Guazzi M, Samaja M, Arena R, Vicenzi M, Guazzi MD: Long-term use of sildenafil in the therapeutic management of heart failure. J Am Coll Cardiol 2007, 50:2136-2144

25. Guazzi M, Vicenzi M, Arena R, Guazzi MD: Pulmonary hypertension in heart failure with preserved ejection fraction: a target of phosphodiesterase-5 inhibition in a 1-year study. Circulation 2011, 124:164-174.

26. Aldashev AA, Kojonazarov BK, Amatov TA, Sooronbaev TM, Mirrakhimov MM, Morrell NW, Wharton J, Wilkins MR: Phosphodiesterase type 5 and high altitude pulmonary hypertension. Thorax 2005, 60:683-687. 
27. Badesch DB, Hill NS, Burgess G, Rubin L, Barst RJ, Galie N, Simonneau G: Sildenafil for pulmonary arterial hypertension associated with connective tissue disease. J Rheumatol 2007, 34:2417-2422.

28. Bharani A, Patel A, Saraf J, Jain A, Mehrotra S, Lunia B: Efficacy and safety of PDE-5 inhibitor tadalafil in pulmonary arterial hypertension. Indian Heart $J$ 2007, 59:323-328.

29. Galie N, Brundage BH, Ghofrani HA, Oudiz RJ, Simonneau G, Safdar Z, Shapiro S, White RJ, Chan M, Beardsworth A, Frumkin L, Barst RJ: Tadalafil therapy for pulmonary arterial hypertension. Circulation 2009, 119:2894-2903.

30. Jing ZC, Yu ZX, Shen JY, Wu BX, Xu KF, Zhu XY, Pan L, Zhang ZL, Liu XQ, Zhang YS, Jiang $X$, Galie N: Vardenafil in pulmonary arterial hypertension: a randomized, double-blind, placebo-controlled study. Am J Respir Crit Care Med 2011, 183:1723-1729.

31. Lewis GD, Shah RV, Pappagianopolas PP, Systrom DM, Semigran MJ: Determinants of ventilatory efficiency in heart failure: the role of right ventricular performance and pulmonary vascular tone. Circ Heart Fail 2008, 1:227-233.

32. Sastry BK, Narasimhan C, Reddy NK, Raju BS: Clinical efficacy of sildenafil in primary pulmonary hypertension: a randomized, placebo-controlled, double-blind, crossover study. J Am Coll Cardio/ 2004, 43:1149-1153.

33. Bocchio M, Pelliccione F, Passaquale G, Mihalca R, Necozione S, Desideri G Francavilla F, Ferri C, Francavilla S: Inhibition of phosphodiesterase type 5 with tadalafil is associated to an improved activity of circulating angiogenic cells in men with cardiovascular risk factors and erectile dysfunction. Atherosclerosis 2008, 196:313-319.

34. Rosano GM, Aversa A, Vitale C, Fabbri A, Fini M, Spera G: Chronic treatment with tadalafil improves endothelial function in men with increased cardiovascular risk. Eur Urol 2005, 47:214-220.

35. van Ahlen H, Wahle K, Kupper W, Yassin A, Reblin T, Neureither M: Safety and efficacy of vardenafil, a selective phosphodiesterase 5 inhibitor, in patients with erectile dysfunction and arterial hypertension treated with multiple antihypertensives. J Sex Med 2005, 2:856-864

36. Goldberg DJ, French B, McBride MG, Marino BS, Mirarchi N, Hanna BD, Wernovsky G, Paridon SM, Rychik J: Impact of oral sildenafil on exercise performance in children and young adults after the fontan operation: a randomized, double-blind, placebo-controlled, crossover trial. Circulation 2011, 123:1185-1193.

37. Goldberg DJ, French B, Szwast AL, McBride MG, Marino BS, Mirarchi N, Hanna BD, Wernovsky G, Paridon SM, Rychik J: Impact of sildenafil on echocardiographic indices of myocardial performance after the Fontan operation. Pediatr Cardiol 2012, 33:689-696.

38. Amin A, Mahmoudi E, Navid H, Chitsazan M: Is chronic sildenafil therapy safe and clinically beneficial in patients with systolic heart failure? Congest Heart Fail 2013, 19:99-103.

39. Guazzi M, Vicenzi M, Arena R: Phosphodiesterase 5 inhibition with sildenafil reverses exercise oscillatory breathing in chronic heart failure: a long-term cardiopulmonary exercise testing placebo-controlled study. Eur J Heart Fail 2012, 14:82-90.

40. Suntharalingam J, Treacy CM, Doughty NJ, Goldsmith K, Soon E, Toshner MR, Sheares KK, Hughes R, Morrell NW, Pepke-Zaba J: Long-term use of sildenafil in inoperable chronic thromboembolic pulmonary hypertension. Chest 2008, 134:229-236.

41. Klingbeil AU, Schneider M, Martus P, Messerli FH, Schmieder RE: A meta-analysis of the effects of treatment on left ventricular mass in essential hypertension. Am J Med 2003, 115:41-46.

42. Tsutamoto T, Wada A, Maeda K, Mabuchi N, Hayashi M, Tsutsui T, Ohnishi M, Sawaki M, Fujii M, Matsumoto T, Matsui T, Kinoshita M: Effect of spironolactone on plasma brain natriuretic peptide and left ventricular remodeling in patients with congestive heart failure. J Am Coll Cardiol 2001, 37:1228-1233.

43. Khouri MG, Peshock RM, Ayers CR, de Lemos JA, Drazner MH: A 4-tiered classification of left ventricular hypertrophy based on left ventricular geometry: the Dallas heart study. Circ Cardiovasc Imaging 2010, 3:164-171.

44. Chatterjee S, Biondi-Zoccai G, Abbate A, D'Ascenzo F, Castagno D, Van Tassell B, Mukherjee D, Lichstein E: Benefits of beta blockers in patients with heart failure and reduced ejection fraction: network meta-analysis. BMJ 2013, 346:f55

45. Pokreisz $P$, Vandenwijngaert $S$, Bito $V$, Van den Bergh $A$, Lenaerts I, Busch $C$, Marsboom G, Gheysens O, Vermeersch P, Biesmans L, Liu X, Gillijns H, Pellens M, Van Lommel A, Buys E, Schoonjans L, Vanhaecke J, Verbeken E, Sipido K, Herijgers P, Bloch KD, Janssens SP: Ventricular phosphodiesterase- 5 expression is increased in patients with advanced heart failure and contributes to adverse ventricular remodeling after myocardial infarction in mice. Circulation 2009, 119:408-416.

46. Zhuang XD, Long M, Li F, Hu X, Liao XX, Du ZM: PDE5 inhibitor sildenafil in the treatment of heart failure: a meta-analysis of randomized controlled trials. Int J Cardiol 2014, 172:581-587.

47. Galasko Gl, Lahiri A, Barnes SC, Collinson P, Senior R: What is the normal range for $\mathrm{N}$-terminal pro-brain natriuretic peptide? How well does this normal range screen for cardiovascular disease? Eur Heart J 2005, 26:2269-2276.

48. Eggers KM, Lagerqvist $B$, Venge $P$, Wallentin L, Lindahl B: Prognostic value of biomarkers during and after non-ST-segment elevation acute coronary syndrome. J Am Coll Cardiol 2009, 54:357-364.

49. Santos RC, de Faria AP, Barbaro NR, Modolo R, Ferreira-Melo SE, Matos-Souza JR, Coelho OR, Yugar-Toledo JC, Fontana V, Calhoun D, Moreno H: Tadalafil-induced improvement in left ventricular diastolic function in resistant hypertension. Eur J Clin Pharmacol 2014, 70:147-154.

50. Borlaug BA, Melenovsky V, Marhin T, Fitzgerald P, Kass DA: Sildenafil inhibits beta-adrenergic-stimulated cardiac contractility in humans. Circulation 2005, 112:2642-2649.

51. McAlister FA, Wiebe N, Ezekowitz JA, Leung AA, Armstrong PW: Metaanalysis: beta-blocker dose, heart rate reduction, and death in patients with heart failure. Ann Intern Med 2009, 150:784-794.

52. Herrmann HC, Chang G, Klugherz BD, Mahoney PD: Hemodynamic effects of sildenafil in men with severe coronary artery disease. $N$ Engl J Med 2000, 34:1622-1626.

53. Wu X, Yang T, Zhou Q, Li S, Huang L: Additional use of a phosphodiesterase 5 inhibitor in patients with pulmonary hypertension secondary to chronic systolic heart failure: a meta-analysis. Eur $J$ Heart Fail 2014, 16:444-453.

54. Isidori AM, Corona G, Aversa A, Gianfrilli D, Jannini EA, Foresta C, Maggi M, Lenzi A: The SIAMS-ED Trial: a national, independent multicentre study on cardiometabolic and hormonal impairment of men with erectile dysfunction treated with vardenafil. Int J Endocrinol 2014, 2014:858715.

55. Jannini EA, Isidori AM, Gravina GL, Aversa A, Balercia G, Bocchio M, Boscaro M, Carani C, Corona G, Fabbri A, Foresta C, Forti G, Francavilla S, Granata AR, Maggi M, Mansani R, Palego P, Spera G, Vetri M, Lenzi A: The ENDOTRIAL study: a spontaneous, open-label, randomized, multicenter, crossover study on the efficacy of sildenafil, tadalafil, and vardenafil in the treatment of erectile dysfunction. J Sex Med 2009, 6:2547-2560.

56. Sasaki H, Nagayama T, Blanton RM, Seo K, Zhang M, Zhu G, Lee DI, Bedja D, Hsu S, Tsukamoto O, Takashima S, Kitakaze M, Mendelsohn ME, Karas RH, Kass DA, Takimoto E: PDE5 inhibitor efficacy is estrogen dependent in female heart disease. J Clin Invest 2014, 124:2464-2471.

57. Williamson P, Clarke M: The COMET (Core Outcome Measures in Effectiveness Trials) Initiative: Its Role in Improving Cochrane Reviews. Cochrane Database Syst Rev 2012, 5:ED000041.

\section{doi:10.1186/s12916-014-0185-3}

Cite this article as: Giannetta et al: Is chronic inhibition of phosphodiesterase type 5 cardioprotective and safe? A meta-analysis of randomized controlled trials. BMC Medicine 2014 12:185.

\section{Submit your next manuscript to BioMed Central and take full advantage of:}

- Convenient online submission

- Thorough peer review

- No space constraints or color figure charges

- Immediate publication on acceptance

- Inclusion in PubMed, CAS, Scopus and Google Scholar

- Research which is freely available for redistribution 